\title{
1 A Notch-dependent transcriptional mechanism controls expression of temporal \\ 2 patterning factors in Drosophila medulla
}

3 Alokananda Ray ${ }^{1}$ and Xin $\mathrm{Li}^{1,2}$

4 1. Department of Cell and Developmental Biology, University of Illinois at Urbana-Champaign,

$5 \quad$ Urbana- IL 61801

$6 \quad$ 2. Corresponding author, email: lixin@illinois.edu

\section{Abstract}

8 Temporal patterning is an important mechanism for generating a great diversity of neuron

9 subtypes from a seemingly homogenous progenitor pool in both vertebrates and invertebrates.

10 Drosophila neuroblasts have been shown to be temporally patterned by sequentially expressed

11 Temporal Transcription Factors (TTFs). These TTFs are proposed to form a transcriptional

cascade based on mutant phenotypes, although direct transcriptional regulation between TTFs

13 has not been verified in most cases. Furthermore, it is not known how the temporal transitions

14 are coupled with generation of the appropriate number of neurons at each stage. We use neuroblasts of the Drosophila optic lobe medulla to address these questions, and show that the expression of TTFs Sloppy-paired $1 / 2$ (SIp1/2) is regulated at transcriptional level directly by

17 two other TTFs and the cell-cycle dependent Notch signaling through two cis-regulatory 
Notch-pathway dependent mechanism through which the cell cycle progression regulates the

21

22

\section{3}

timing of a temporal transition within a TTF transcriptional cascade.

\section{Introduction}

Generation of a great diversity of neurons from a small pool of neural progenitors is critical for constructing functional nervous systems. This is achieved in part by integration of temporal patterning and spatial patterning of neural progenitors [reviewed in ${ }^{1-4}$ ]. Temporal patterning of neural progenitors refers to the generation of differently fated progeny in a birth-order dependent manner, and this is observed in both invertebrates and vertebrates [reviewed in ${ }^{5-7}$

]. The central nervous system in Drosophila melanogaster has been an excellent model to study temporal patterning. Neural progenitors called neuroblasts (NBs) have been shown to sequentially express series of temporal transcription factors (TTFs), which are each required to specify subsets of neuron fates [reviewed in ${ }^{8,9}$ ]. For example, neuroblasts in the embryonic ventral nerve cord (vnc) are temporally patterned by a TTF cascade Hunchback ( $\mathrm{Hb})$, Kruppel (Kr), Nubbin/Pdm2 (Pdm), Castor (Cas) and Grainy head (Grh) ${ }^{10-13}$, while Drosophila optic lobe medulla neuroblasts utilize a different TTF cascade composed of Homothorax (Hth), SoxNeuro (SoxN), Doublesex-Mab related 99B (Dmrt99B), Odd paired (Opa), Eyeless (Ey), Earmuff (Erm), Homeobrain (Hbn), Sloppy-paired (SIp1,SIp2), Scarecrow (Scro), Dichaete (D), BarH1/2, Tailless

(TII), and Glial cell missing $(\mathrm{Gcm})^{14-19}$. In these TTF cascades, cross-regulations were identified among TTF genes based on loss and gain of function phenotypes, and they were proposed to 40 form a transcriptional cascade ${ }^{11-13,}{ }^{14-19,20}$ that can in theory self-propagate ${ }^{21}$. However, with a 
41 few exceptions ${ }^{22}$, the cis-regulatory elements of these TTF genes haven't been characterized,

42 and thus it is not known whether these cross-regulations are indeed direct transcriptional

43 regulations. Moreover, in the embryonic TTF cascade, although mis-expression of one TTF is

44 sufficient to activate the next TTF and repress the "next plus one" TTF, loss of one TTF often

45 does not block temporal progression, but only causes the corresponding fates to be skipped ${ }^{11}$.

46 In the medulla TTF cascade, one TTF is required but not sufficient to promote the TTF cascade

47 progression ${ }^{19}$. These studies suggest that additional mechanisms in addition to cross-

48 regulations among TTFs might function to regulate TTF progression.

50 As the neural progenitors go through the TTF cascade, they need to generate a certain number

51 of postmitotic progeny as each temporal stage. Therefore, it has been questioned whether cell

52 cycle progression is required for the TTF cascade progression. In the embryonic VNC TTF

53 cascade, cytokinesis is required for the first transition ( $\mathrm{Hb}$ to $\mathrm{Kr}$ ) by promoting the nuclear

54 export of seven-up (svp) mRNA encoding a switching factor, but all later temporal transitions

55 progress normally in G2-arrested neuroblasts ${ }^{11,23,24}$. In larval VNC NBs, timely transition from

56 the Imp /Castor/Chinmo to Syncrip/Broad stage also requires cell cycle progression ${ }^{25}$. These

57 results suggest that the requirement of cell-cycle progression varies depending on the specific

58 temporal transition. In vertebrate cortical progenitors, the temporal transition from generating

59 deep layer neurons to generating upper layer neurons is not affected by blocking cell cycle

60 progression when constitutively active Notch signaling is provided ${ }^{26}$. However, it is still

61 possible that Notch signaling might be promoting the temporal transition in the absence of cell

62 cycle progression. 
64 Notch signaling plays pleiotropic roles in neurogenesis and neural development in both

65 vertebrates and invertebrates [reviewed in ${ }^{27}$. Upon binding to a ligand from a neighboring

66 cell, the Notch receptor is cleaved to release the Notch intracellular domain (NICD), which then

67 enters the nucleus and associates with the DNA-binding protein CSL (CBF1/RBPjk/Su(H)/Lag-1).

68 The NICD-CSL then recruits transcriptional coactivator Mastermind (MAM) to activate

69 transcription of Notch target genes, mainly the bHLH transcriptional repressors of the HES

70 (hairy and enhancer of split) /HEY families [reviewed in ${ }^{28-30}$ ]. Drosophila NBs divide

71 asymmetrically multiple times and at each division generate a self-renewed neuroblast and a

72 more differentiated progeny, that is either an intermediate progenitor [Ganglion Mother Cell

73 (GMC) for type I NBs, or Intermediate Neural Progenitor (INP) for type II NBs], or a postmitotic

74 progeny (type 0 NBs) [reviewed in ${ }^{31}$ ]. During the asymmetric division of Drosophila

75 neuroblasts, Numb, a negative regulator of Notch signaling, is asymmetrically localized to the

76 intermediate progenitor, resulting in unidirectional Delta-Notch signaling from the intermediate

77 progenitor to the neuroblast ${ }^{32-35}$. Ectopic activation of Notch signaling in the progeny causes

78 them to revert back into neuroblasts and over-proliferate, while inhibition of Notch signaling in

79 type II NBs eliminated the whole lineage ${ }^{36-39}$. In contrast, in most type I NB lineages, loss of N

80 signaling does not affect neuroblast maintenance, and it was shown that $\mathrm{E}(\mathrm{spl})$ complex

81 proteins and Deadpan act redundantly to maintain the un-differentiated state (stemness) of

82 neuroblasts by repressing differentiation genes ${ }^{39-41}$. Notch signaling is also involved in

83 controlling the daughter cell proliferation modes specifically the type I to type 0 switch in

84 embryonic VNC NBs ${ }^{42,43}$. Work in vertebrate central nervous system suggest that during the 
asymmetric division of neural progenitors, the more differentiated progeny expresses Delta and activates Notch signaling in the sister cell to maintain the neural progenitor fate [reviewed in

$87^{27}$ ]. In addition, Notch signaling show cell cycle dependent activation and the expression of Notch target Hes genes oscillates during the cell cycle in neural progenitors [reviewed in ${ }^{44}$ ]. Although Notch signaling has been shown to play many important roles in neural lineage development, whether Notch signaling also has a role in temporal transitions between TTFs has not been investigated. Here, we use Drosophila medulla neuroblasts to address these questions. In the larval optic lobe, a neurogenesis wave spreads in a medial to lateral direction to sequentially convert neuroepithelial cells into neuroblasts. As a result, medulla neuroblasts of different ages are orderly aligned on the lateral to medial spatial axis with the youngest on the lateral side (Fig. 1A). Medulla neuroblasts divide asymmetrically multiple times to self-renew and to generate a series of GMCs and at the same time they transit through the TTF sequence (Fig. 1A). Among the TTFs, SIp1 and SIp2 are two homologous fork-head family transcription factors expressed in the same temporal pattern in medulla neuroblasts (Fig. 1B-B'"'). In this work, we identified two cis-regulatory enhancer elements that regulate the temporal expression pattern of SIp $1 / 2$, and showed that the previous TTF Ey, another TTF Scro, and the Notch signaling pathway directly regulate the transcription of $s / p$ through these two enhancers. SIp1/2 expression is delayed when $\mathrm{N}$ signaling is lost or when cell cycle progression is blocked which also causes loss of $\mathrm{N}$ 104 signaling. Furthermore, we show that supplying transcriptionally active Notch can rescue the 105 timely Ey to Slp transition in cell-cycle arrested neuroblasts. Thus, our work demonstrates that 
cell-cycle dependent Notch signaling cooperates with TTFs to promote the progression of the

107 TTF transcriptional cascade.

\section{Results}

Expression of SIp1 and SIp2 in medulla neuroblasts is regulated at the level of transcription by

\section{coordinated function of at least two enhancers.}

112 The observed distribution of temporal patterning transcription factors may be regulated at

113 either the transcriptional or post-transcriptional level, and the protein expression pattern is not necessarily the same as that of the mRNA transcripts. A case in point is the expression pattern

117 distributed in neuroblasts, SoxN protein is only expressed in the youngest neuroblasts,

118 suggesting that post-transcriptional mechanisms are at work to confine the actual domain of

119 SoxN protein expression ${ }^{17}$. To examine whether the expression of SIp1/2 in medulla

120 neuroblasts is regulated at transcription, we observed the distributions of s/p1 and s/p2 mRNA

121 transcripts by fluorescence in situ-hybridization (Fig. 1C- $C^{\prime \prime \prime}$ ). The observed patterns of s/p1 and

122 slp2 mRNA expression closely parallel the expression of SIp1 and SIp2 proteins (Fig. 1B-B'"'), and

123 are also corroborated by scRNA-seq data ${ }^{17}$, indicating that the expression of $\operatorname{s} / p 1$ and $\operatorname{s} / p 2$ is

124 directly regulated at transcription.

125 We then sought to identify transcriptional enhancers that enable expression of the s/p genes in

126 the characteristic pattern observed in medulla neuroblasts. From our examination of expression 
127 patterns of GFP in third instar larval brains driven by Gal4 lines under the regulation of putative

128 enhancer elements ${ }^{45-47}$ (available from the Janelia FlyLight image database

129 https://flweb.janelia.org/cgi-bin/flew.cgi), we identified the GMR35H02 Gal4 line as containing a

130 candidate enhancer driving SIp1/2 expression in medulla neuroblasts. We then verified the

131 expression of UAS-GFP driven by GMR35H02-Gal4 and found that indeed the GFP expression in

132 medulla neuroblasts was initiated at the same time as endogenous SIp1 and SIp2

133 (Supplementary Fig. S1A-B'). Subsequently, we cloned the $3.75 \mathrm{kbp}$ element corresponding to

134 the GMR35H02 Gal4 regulatory sequence upstream of a EGFP reporter gene with a basal hsp70

135 promoter in the vector pJR12 ${ }^{48}$ and constructed transgenic lines. In these lines GFP was

136 expressed in the same pattern as the GMR35H02Gal4 driving UASGFP. Through successive

137 iterations of enhancer-bashing experiments whereby we cloned progressively smaller segments

138 of the initial $3.75 \mathrm{kbp}$ element (See Supplementary Table 1 for primer sequences used) in the

139 above-described vector and observed GFP reporter expression in transgenic flies, we identified

140 a $220 \mathrm{bp}$ fragment within the larger GMR35H02 regulatory DNA which preserved the expression

141 pattern of the GFP reporter (Fig. 1E-E'"') as that of UAS-GFP driven by the GMR35H02 -Gal4.

142 This we reasoned is a minimal enhancer driving expression of Slp genes in medulla neuroblasts.

143 Deletion of this element by CRISPR-Cas9 however failed to completely eliminate the expression

144 of SIp1 and SIp2 in neuroblasts (Supplementary Fig. S1C-C'"'), though it noticeably reduced the

145 expression domain of SIp1 (Supplementary Fig. S1E). Given the likelihood of developmentally

146 regulated gene expression being controlled by multiple enhancers ${ }^{49,50}$ and the importance of

147 enhancer redundancy to achieving robust developmental outcomes ${ }^{49,51,52}$, this hinted that

148 other enhancers may act redundantly to regulate SIp1/2 expression in medulla neuroblasts. We 
149 identified other potential enhancers of SIp1/2 expression from the REDfly database ${ }^{53,54}$ and

150 tested their roles in driving SIp1/2 expression in the context of medulla neuroblasts. We

151 screened available transgenic lines expressing GFP or lacZ reporters driven by putative

152 enhancer elements (kind gifts from Dr. Miki Fujioka and Dr. James B. Jaynes) and cloned other

153 candidate regulatory segments in the pJR12 GFP-reporter vector to compare GFP transgene

154 expression to endogenous SIp1/2 expression. Through this screen we identified a second

155 regulatory segment corresponding to the REDfly enhancer d5778 (Fig. 1D) that can drive GFP

156 expression in medulla neuroblasts within the endogenous SIp1/2 expression domain.

157 Subsequently we found that an $850 \mathrm{bp}$ segment within the d5778 enhancer is sufficient to

158 initiate GFP expression in this pattern (Fig. 1F-F"'). Reporter expression driven by this second

159 enhancer (henceforth referred to as d5778 850bp enhancer) is initiated at a slightly later time

160 compared to the first identified enhancer, in older Slp expressing neuroblasts and also in glial

161 cells at the junction of the medulla and the central brain. Our analysis of the REDfly enhancers

162 that supposedly regulate expression of SIp1/2 also revealed that the earlier identified $220 \mathrm{bp}$

163 enhancer segment is contained within another REDfly enhancer named u8772 (Fig. 1D). Thus,

164 we refer to the first identified enhancer henceforth as u8772 $220 \mathrm{bp}$. As in case of the u8772

$165220 \mathrm{bp}$ enhancer deletion, sole deletion of d5778 enhancer by CRISPR-Cas9 again did not abolish

166 Slp1/2 expression(Supplementary Fig. S1D- $D^{\prime \prime \prime}$ ), suggesting that these enhancers function

167 redundantly and may make additive contributions to attaining the observed levels of SIp1 and

168 Slp2 expression.

169 Bioinformatic analyses of the sequences of our identified enhancers (see Methods) identified

170 binding sites for the previous TTF Ey, the CSL transcription factor Su(H), Slp1 itself, and a Nk2- 
171 class homeodomain transcription factor ventral nervous system defective (vnd) (Supplementary

172 Table 2). The previous TTF Ey has been shown to be required but not sufficient for activating Slp

173 genes' expression in medulla neuroblasts, but it was not known whether this regulation is

174 direct. Vnd is not expressed in medulla neuroblasts according to our single-cell RNA-sequencing

175 data $^{17}$, but a related homologous NK-2 homeobox transcription factor Scarecrow ${ }^{55}$ (Scro) is

176 expressed around the same time as SIp, and loss of Scro leads to significantly reduced SIp

177 expression level ${ }^{17}$. The CSL transcription factor $\mathrm{Su}(\mathrm{H})$ is the Drosophila homolog of RBP-J ${ }^{56,57}$,

178 and the key DNA-binding component of the active Notch transcriptional complex. The Notch

179 signaling pathway plays an overarching role as a master regulator of development and

180 differentiation in neuroblasts, but it hasn't been shown to regulate the expression of temporal

181 patterning genes in neural progenitors. Therefore, we next tested whether SIp1/2 expression is

182 regulated by Notch signaling.

The Notch pathway regulates SIp1 and SIp2 expression in medulla neuroblasts

184 We observed expression of SIp1 and SIp2 in RNAi knockdown clones of key Notch pathway

185 components induced by ayGal4 (actin>FRT-y+-STOP-FRT-Gal4, in which actin promoter drives

186 Gal4 expression only after a STOP cassette is excised by the action of heat shock activated

187 Flippase $\left.{ }^{58}\right)$. Since SIp1 and SIp2 are two homologous genes located in proximity ${ }^{59}$ and

preceding that of SIp2 expression (Fig. 1B- $\mathrm{B}^{\prime \prime \prime}$ ), in all subsequent figures we have shown data for 
delayed within the RNAi knockdown clones marked by GFP expression (Fig. 2A-A'"', B-B'"' respectively) compared to wild type neuroblasts of the same age (i.e., in the same vertical 'stripe'). A similar effect is seen when dominant negative variant of the third core component of the Notch transcriptional complex mastermind (mam) is expressed under the control ayGal4

(Fig. 2C-C'"'). These data suggest that timing of SIp1/2 expression in medulla neuroblasts is regulated by the Notch pathway.

To identify the cognate ligand of Notch that functions in activating the Notch signaling in medulla neuroblasts, we examined expression of known Notch ligands- Delta ${ }^{61}$, Serrate ${ }^{62,63}$ and

207 2D-D'") confirming this expectation.

\section{Notch pathway and Ey regulate the activity of s/p enhancers.}

Since $\mathrm{Su}(\mathrm{H})$ and Ey are required for SIp expression, we next tested whether these regulations

212 factors we had identified binding sites for bound to the enhancers and enabled them to initiate 
214 function clones of these transcription factors. We induced RNAi knockdown clones for Ey and

$215 \mathrm{Su}(\mathrm{H})$ using ayGal4 in transgenic flies expressing wild type GFP reporters driven by these two

216 enhancers and compared the expression of GFP in wild type neuroblasts and in neuroblasts

217 where each of these TFs had been individually knocked down.

218 For both enhancers, GFP reporter expression was lost in eyRNAi clones (Fig 3A-B') confirming an

219 earlier observation that Ey was required for initiating SIp1/2 expression. It also strengthened

220 the evidence that these two were indeed bona-fide enhancers activating SIp1/2 expression.

221 Similarly, GFP reporter expression was lost or delayed in su(H) RNAi clones for both enhancers

222 (Fig 3C-D'), confirming that $\mathrm{Su}(\mathrm{H}) / \mathrm{N}$ pathway regulates Slp1/2 expression through these two

223 enhancers. Since SIp binding sites were also identified in the s/p enhancers, we next tested the

224 effect of loss of SIp1/2 function on the expression of our GFP reporters. To this end we induced

225 slp1/2 loss of function (LOF) mutant clones in flies carrying the GFP reporter and UbiRFP

226 FRT4OA. In Fig. 3E-F' s/p LOF clones are visible as dark regions devoid of RFP whereas wild type

227 neuroblasts are marked by RFP expression from the Ubiquitin promoter. Reporter expression

228 driven by both enhancers within s/p LOF neuroblasts is normal as in wild type neuroblasts.

229 Though this suggests that SIp1/2 are not required to initiate their own expression, it does not

230 preclude a possible role of SIp1/2 in sustaining their own expression in older SIp1/2 stage

231 neuroblasts or progeny, but the perdurance of the GFP reporter prevents us from testing this

232 possible role. In summary, our genetic experiments confirmed that identified enhancers of

233 s/p1/2 respond to activation by Ey and $\mathrm{Su}(\mathrm{H})$.

234 Mutations of potential binding sites for $\mathrm{Ey}, \mathrm{Su}(\mathrm{H})$ and Scro in s/p1/2 enhancers impair 
To further test whether the regulators we identified indeed regulate SIp1/2 expression directly through binding to the enhancers, we set out to mutate all possible binding sites for each of the regulators- Ey, Su(H), Scro and SIp1, on each enhancer. We identified all potential matches to consensus binding motifs for these factors using the DNA Pattern Recognition Tool within the Sequence Manipulation Suite ${ }^{65}$. Custom-designed gene blocks containing the mutated enhancer sequences were cloned upstream of the GFP reporter in pJR12 vector as before, and

242 transgenic flies expressing these reporter constructs were then made (Supplementary Table1).

243 We next compared the expression patterns and intensities of GFP reporters with mutated TF

244 binding sites against GFP reporter expression driven by the 'wild type' version of that same 245 enhancer (Fig. 4A-A'"', Fig. 5A-A'"'). To compare GFP reporter intensities between wild type and 246 mutated variants of an enhancer, identical microscope illumination and imaging settings were 247 used for all variants of the same enhancer. $C^{\prime \prime \prime}, F$ and Fig. 5D-D'"',F). For the d5778 850 bp enhancer, the expression of GFP in glial cells is still present and likely intensified when $\mathrm{Su}(\mathrm{H})$ binding sites are mutated. Since it is impossible to

252 avoid contributions to the GFP signal intensity from surface-glial cells in neuroblast stacks we 253 have quantified, the GFP signal intensity reflected in our measurements for the d5778 Su(H) 254 site mutant reporter seems high and also very variable (Fig. 4F). However, GFP expression from 255 the $\mathrm{d} 5778 \mathrm{Su}(\mathrm{H})$ site mutated reporter in neuroblasts alone is dramatically reduced compared 256 to the wild type. Mutation of scro sites in the $48772220 \mathrm{bp}$ enhancer also led to a loss of GFP 257 expression (Fig. 4D-D'"',F), whereas GFP expression was considerably weakened in the d5778 
850bp scro site mutant enhancer (Fig. 5B-B"', F). In contrast, mutation of SIp1 binding sites did not significantly affect the reporter expression (Fig. 4E-E'", F, and Fig. 5E-E'”'). In summary, our results provided strong evidence that Ey, Su(H) and Scro directly regulate SIp1/2 expression through binding to its two enhancers.

\section{DamID-sequencing confirms binding of Ey and Su(H) to identified enhancers of SIp1/2.}

DamID-sequencing can be used to profile genome-wide binding patterns for transcription factors and chromatin proteins ${ }^{66,67}$. We used a SoxNGal4 that is expressed in all medulla neuroblasts ${ }^{17}$ to drive the expression of UAS-DamSu(H) or UAS-DamEy fusion proteins, and inferred the genome-wide binding patterns of Ey and $\mathrm{Su}(\mathrm{H})$ through DamID-sequencing. For each transcription factor two biological replicates were sequenced. Reproducible peaks of Ey and $\mathrm{Su}(\mathrm{H})$ binding were observed in both replicates in the genomic region corresponding to the identified enhancers of SIp1/2 transcription (Fig. 6A). Additionally, we also observed peaks of Ey and $\mathrm{Su}(\mathrm{H})$ binding in the genomic region of scro gene (Supplementary Fig. S2A). It has been reported that Scro expression is initiated in medulla neuroblasts at about the same time as SIp1/2. This raises the possibility that Scro expression may also be initiated by either Ey, $\mathrm{Su}(\mathrm{H})$ or both. In another temporal gene homeobrain $(h b n)$ that is turned on in the Ey stage ${ }^{16,18}$, Ey and $\mathrm{Su}(\mathrm{H})$ binding peaks were also observed (Supplementary Fig. S2B). Genome-wide about
$62.78 \%$ of all $\mathrm{Su}(\mathrm{H})$ bound genes and $60.3 \%$ of all Ey bound genes were bound by both Ey and

$\mathrm{Su}(\mathrm{H})$ (Fig. 6B). Enriched GO terms for these co-bound genes include: neuroblast fate determination, cell division, asymmetric cell division, apical cell polarity, Notch signaling pathway and axon guidance (Fig 6C-H). Taken together these data support evidence from our 
279

280

281

282

283

284

285

286

287

288

289

290

291

292

293

294

295

296

297

298

299

genetics studies and together they suggest that Ey and $\mathrm{Su}(\mathrm{H}) / \mathrm{N}$ pathway activate $\mathrm{Slp} 1 / 2$ transcription by direct binding to SIp1/2 enhancers.

\section{Notch signaling is dependent on cell-cycle progression.}

Although it has been well-established that GMCs generated by the asymmetric division of neuroblasts signal to their sister neuroblasts to provide the Notch signaling ${ }^{32,33}$, it hasn't been demonstrated whether Notch signaling is lost in cell-cycle arrested medulla neuroblasts since they fail to generate GMCs. We used a regional Gal4, vsxGal4 that is expressed in the center domain of the medulla crescent starting in the neuroepithelium ${ }^{68}$, to drive UAS-DCR2 and a RNAi line against Proliferating Cell Nuclear Antigen (PCNA), which is essential for DNA replication and S phase progression ${ }^{69}$. Blocking cell cycle progression caused premature transformation of neuroepithelial cells to neuroblasts, as has been previously reported ${ }^{70}$. These neuroblasts express the normal neuroblast marker Deadpan (Dpn) (Fig. 7A-A"), but produce few progeny, and some neuroblasts are present in the deep layers which would normally be occupied by neuronal progeny. A large fraction of these neuroblasts in the central domain don't express $\mathrm{E}(\mathrm{spl})$ mpGFP, a Notch signaling reporter ${ }^{61}$, while all neuroblasts in the control regions express $\mathrm{E}(\mathrm{spl})$ myGFP (Fig. 7A-A"). Thus, Notch signaling is largely lost in cell-cycle arrested neuroblasts.

\section{The role of cell cycle progression in the medulla TTF cascade.}

Next, we tested whether blocking cell-cycle progression also affected the medulla TTF cascade progression. In vsxGal4>PCNA RNAi brains, neuroblasts in the affected region do not express either Ey or SIp1/2, but keep expressing SoxN (Fig. 7B-C'). This suggests that an earlier 
301 temporal transition step is blocked which is required for Ey expression, and this prevents us

302 from examining whether the Ey to Slp transition also requires cell cycle progression. To

303 circumvent this problem, we used the ayGal4 to drive UAS-DCR2 and PCNA RNAi. By adjusting

304 heat shock timing to induce clones, we were able to obtain a condition where Ey expression is

305 minimally affected, and at this condition we observed that SIp2 expression is still severely

306 delayed (Fig. 7E-E'"'). In addition to the timing of clone induction, the ayGal4 is also likely to be

307 weaker than VsxGal4 at inducing RNAi, and the cell cycle is likely only slowed down rather than

308 arrested when using ayGal4, because there are still some progeny produced in such clones.

309 Furthermore, SIp1/2 expression is also delayed when we knock down String /Cdc25 required for

310 the $\mathrm{G} 2$ to $\mathrm{M}$ phase transition ${ }^{71}$, or overexpress Dacapo (Dap, a Cyclin-dependent kinase

311 inhibitor in the CIP/KIP family) ${ }^{72}$ using ayGal4 (Supplementary Figure S3A-B'"). Thus, cell cycle

312 progression is also required for the precise timing of Ey to Slp1/2 transition.

314 Notch signaling can rescue Slp expression delay caused by cell cycle defects.

315 Finally, we tested if supplying Notch signaling to cell-cycle arrested or delayed neuroblasts can

316 rescue the timing of SIp expression initiation. We supplied active Notch in neuroblasts using a

$317 d p n>F R T$-stop-FRT3-FRT-NICD transgene (which drives the expression of NICD from the $d p n$

318 enhancer in presence of heat shock activated Flippase; see Methods), while simultaneously

319 inducing knockdown of PCNA using ayGal4 under the same heat shock condition that leaves Ey

320 expression largely unaffected. Supplying NICD was sufficient to rescue SIp2 expression in PCNA

321 RNAi clones (Fig. 7E-E'”'). This suggests that for the Ey to Slp transition, the presence of active

322 Notch signaling can substitute for the requirement for the cell-cycle progression. 


\section{Discussion}

325 Evidence of direct transcriptional activation in the medulla TTF cascade.

326

327

328

329

330 expression level ${ }^{17}$. Mutation of most probable Scro binding sites on the u8772 220bp enhancer

Drosophila neuroblasts are temporally patterned by sequentially expressed TTFs. Although the expression pattern and mutant phenotypes suggest that TTFs form a transcriptional cascade, direct transcriptional regulation between TTFs has not been demonstrated in most cases. This work has characterized two enhancers of the s/p genes that enable the expression of SIp1 and SIp2 in medulla neuroblasts. The u8772 $220 \mathrm{bp}$ enhancer is activated at an earlier stage relative to the d5778 850bp enhancer. The d5778 850bp enhancer also seems to drive SIp2 expression in surface glial cells ensheathing the medulla neuroblasts. In these two enhancers, we identified sites for the previous TTF -Ey and Scro-a TTF expressed at around the same time as SIp1 $1^{16,17}$. Deleting either enhancer alone did not eliminate the expression of endogenous SIp1 and SIp2, suggesting that they act redundantly with one another and possibly with other enhancers active in this context. Using GFP reporter assays, we have shown that mutation of Ey binding sites in these enhancers abolishes reporter expression similar to genetic experiments where we observe a loss of GFP reporter within ey RNAi clones. Our results are also consistent with previous studies that showed a complete loss of endogenous SIp1/2 expression in UAS-ey-RNAi expressing neuroblasts ${ }^{19}$. We also confirmed the in vivo binding of Ey to the identified enhancers of SIp by Dam-ID sequencing. The expression of the TTF Scro is initiated simultaneously as SIp1/2, and it has been shown that loss of Scro significantly reduces SIp led to a complete loss of GFP reporter expression. In contrast, a similar manipulation of the 
d5778 850bp enhancer led to a decreased intensity of GFP reporter expression but did not

347 eliminate it. Thus the combined effect of mutating Scro binding sites on both enhancers recapitulates the observed impact of Scro knock-down on endogenous Slp1/2 expression, which

349 is reduced expression of SIp1/2 in neuroblasts expressing UAS scro-RNAi and a consequent loss of neural fates specified by SIp1/2 in their progeny. The observed differences in effects of Scro binding site mutation on the two enhancers also highlights a general feature of enhancer redundancy in fine-tuning expression of developmentally regulated genes- that different enhancers are often sensitive to different environmental signals ${ }^{49}$, which ultimately helps in

354 ensuring robust gene expression if an important regulator is absent or is present but at

355 insufficient concentrations for enhancer activation. It is also interesting to note that our 356 observation of multiple enhancers regulating SIp1/2 expression is consistent with regulation of

357 Slp1/2 in other developmental contexts. Previous studies have noted the presence of multiple 358 enhancers of SIp1/2 expression in the vicinity of the $\operatorname{s} / p 1$ and $\operatorname{s} / p 2$ coding loci. Many of these 359 regulatory DNA segments function as stripe enhancers enabling SIp1/2 to function as pair-rule genes during embryonic segmentation ${ }^{50}$. Though these enhancers share some overlapping

361 functions and domains of activation, a full complement of stripe enhancers is required for 362 maintaining parasegment boundaries and wingless expression ${ }^{74,50}$. expression to ensure the sufficient duration of the Ey window. How is the timing controlled? 
From our analyses of the s/p1/2 enhancer sequences we found several binding sites for the CSL

371 involvement of the Notch pathway in regulating SIp1/2, we observed effects of knocking down

372 key Notch pathway components on endogenous SIp1/2 expression. In all cases we observed a

373 delay in expression of SIp1/2 in neuroblasts expressing the RNAi knockdowns compared to the

374 time of SIp1/2 initiation in wild type neuroblasts. Mutating $\mathrm{Su}(\mathrm{H})$ binding sites in the $u 8772$

$375220 \mathrm{bp}$ and the d5778 850bp enhancers led to a loss or delay of GFP reporter expression in

376 neuroblasts. These results suggest that the $48772220 \mathrm{bp}$ and the $\mathrm{d} 5778850 \mathrm{bp}$ enhancers are

377 sensitive to $\mathrm{Su}(\mathrm{H})$ and further support the role of the Notch pathway in regulating the timing of

378 SIp1/2 expression. However, Ey may still play a more central role in activating SIp1/2 expression

379 than the Notch pathway since SIp1/2 are still expressed albeit later in the absence of Su(H) and other Notch components. As with Ey, we confirmed $\mathrm{Su}(\mathrm{H})$ binding to our enhancers of interest

using DamID-seq. Thus, our work provided strong evidence that $\mathrm{N}$ signaling, a general signaling pathway involved in neuroblast development, regulates the timing of activation of a TTF gene.

This might be part of mechanisms to promote TTF cascade progression specifically in neuroblasts, where Notch signaling is active.

387 What might explain the delay in SIp1/2 expression in the absence of Notch function? Recent 388 developments of single-molecule Fluorescence In Situ Hybridization (smFISH) technology and 
process in molecular detail. Imaging transcription driven by Notch responsive enhancers in

native contexts has shown this process to be inherently 'bursty', i.e., episodes of transcription

(enhancer 'On' state) are punctuated with gaps in activity (enhancer in 'Off' state) ${ }^{75,76}$. The

dosage of NICD modulates the duration of the 'On' phase in one context studied by live imaging

75,76. Additionally, binding of tissue-specific regional factors to these Notch responsive

enhancers may prime these enhancers and help synchronize transcription and sustain a steady

transcriptional output upon Notch binding to enhancers; this helps integrate important

positional cues and the perception of context ${ }^{75}$. Applying these insights to our system, we

suggest that the delay observed may correspond to an increased number of abortive

transcription events in the absence of $\mathrm{Su}(\mathrm{H})$ and Notch and in the presence of Ey alone. Ey may

also act by priming our Notch responsive enhancers providing crucial contextual information

regulation is supported by observations from our DamID data that $62.78 \%$ of $\mathrm{Su}(\mathrm{H})$ bound

407 The role of cell cycle progression and Notch signaling in the medulla TTF cascade 
412 the medulla neuroblasts, we also observed that in $\mathrm{Su}(\mathrm{H})$ mutant clones, the clone size and

413 neuroblast prolifeation is not significantly affected. On the other hand, we observed that $\mathrm{N}$

414 signaling is dependent on cell-cycle progression, and $\mathrm{N}$ target gene is lost when cell cycle

415 progression is blocked.

416 In the medulla, blocking cell cycle progression in neuroepithelial cells prematurely transform

417 them into neuroblasts, and these neuroblasts seem to be arrested in the TTF cascade at the

418 SoxN stage. The expression of Ey and Slp are never turned on in such neuroblasts. When we

419 arrested or slowed down the cell cycle later in neuroblasts to preserve Ey expression, we

420 observed SIp expression is still delayed. Therefore, cell cycle progression also has a role in the

421 Ey to Slp transition. Further we showed that supplying Notch signaling is sufficient to rescue the

422 delay in the Ey to Slp transition caused by cell cycle defect. Thus at the Ey to SIp transition, the

423 cell cycle effect is mediated through the direct regulation of Slp transcription by Notch

424 signaling. Taken together, our results suggest that in Ey stage neuroblasts, Ey is required to

425 initiate Slp expression but not sufficient to activate it to a strong level right away, and after

426 each asymmetric division, activation of Notch signaling in the neuroblast enhances Slp

427 expression, until SIp expression reaches a certain level to repress Ey expression and make the

428 transition. This can be part of a mechanism to coordinate the TTF temporal transition with the

429 cell cycle progression in order to generate the appropriate number of neural progeny at a given

430 temporal stage.

\section{$431 \quad$ Methods}

\section{$432 \quad$ Fly stocks and genetics}


433 Flies were reared on yeast food at $25^{\circ} \mathrm{C}$ unless otherwise stated.

434 Enhancer identification- Flies carrying the GMR35H02-Gal4 insertion (BDSC 49923) were

435 crossed with transgenic flies expressing UAS GFPnIs (BDSC 4776). GFP driven by the GMR35H02-

436 Gal4 was then compared to endogenous SIp1 and SIp2 expression. Procedure for making

437 transgenic constructs and strains is described separately.

438 RNAi experiments- The following RNAi lines were used for RNAi knockdown experiments (In Fig

2); UAS-ey-RNAi (BDSC 32486), UAS-scro ${ }^{R N A i}$ (BDSC 33890), UAS-N-RNAi (BDSC 7078), UAS-

SU(H)-RNAi (VDRC 103597), UAS-DI-RNAi (VDRC 32788), UAS-PCNA-RNAi (VDRC 51253), UAS-

441 stg-RNAi (VDRC 17760).

ayGal4 (actin>FRT-y+-STOP-FRT-Gal4, in which actin promoter drives Gal4 expression after a

STOP cassette is excised by the action of heat shock activated Flippase) was used to drive RNAi

444 of ey, scro and su(h). Flies of genotype ywhsFlp; ayGal4>UAS GFP; UAS Dcr2/Tm6B were crossed

445 to the RNAi lines. Larvae were heat shocked at $37^{\circ} \mathrm{C}$ for 10 minutes 48 hours after egg laying

446 and then raised at $29^{\circ} \mathrm{C}$, until brains of third instar larvae were dissected and stained.

447 For observing effect of ey RNAi on the GFP reporter expression (Fig 3) flies of genotype ywhsFlp;

448 ayGal4>UAS lacZ; eyRNAi (made by combining ayGal4>UAS lacZ on chromosome II (BDSC 4410)

449 with UAS-ey RNAi on chromosome III) were crossed to flies with genotype ywhsFlp; $S p / C y O$;

$450 \quad$ u8772 220bp>GFP (enhancer1) or ywhsFlp; Sp/CyO; d5778 870bp>GFP (enhancer2).

451 For studying effects of Su(H) RNAi on GFP reporter expression, flies of genotype ywhsFlp;

452 ayGal4>lacZ; Tm2/Tm6B were crossed to flies of genotype Dcr2; Su(H) RNAi; u8772 220>GFP

453 (enhancer 1) or Dcr2; Su(H) RNAi; d5778 870> GFP (enhancer 2). 
454 Heat shock protocols for RNAi experiments driven by ayGal4 in presence of GFP reporters were

455 the same as described above for ayGal4 driven RNAi by themselves (i.e., without GFP

456 reporters).

457 Cell cycle arrest experiments- For some experiments examining effect of cell cycle arrest on

458 SIp1/2 expression and on expression of the Notch reporter E(spl)-my::GFP, VsxGal4 was used to

459 drive the expression of UAS PCNA RNAi and UAS Dcr2 in neuroblasts. Flies of genotype VsxG4;

$460 E(s p l)-m y G F P ; U A S$ Dcr2/Tm6B were crossed to those with genotype ywhsFlp; UAS-PCNA RNAi.

461 Larvae were shifted to $29^{\circ} \mathrm{C} 48$ hours after egg laying. Brains of third instar larvae were

462 observed. For other experiments, flies of genotype ywhsFlp; UAS-PCNA RNAi were crossed to

463 flies of genotype ywhsFlp; ayGal4>UAS GFP; UAS Dcr2/Tm6B. Larvae with genotype ywhsFLP;

ayGal4UASGFP /UAS-PCNARNAi; UASDCR2/+ were heat shocked for 8 minutes at $37^{\circ} \mathrm{C} 50$ hours

465 after egg laying (70 hours before they develop into climbing third instar larvae) and were then

466 dissected. For rescuing Notch expression in cell cycle arrested neuroblasts flies of genotype

467 ywhsFlp; ayGal4>UAS GFP; UAS Dcr2/Tm6B were crossed to those with genotype ywhsFlp; UAS

468 PCNA RNAi; dpn>hsNICD. Larvae of genotype ywhsFLP; ayGal4UASGFP/UAS-PCNARNAi;

UASDCR2/dpn>hsNICD were then heat shocked for 8 minutes at $37^{\circ} \mathrm{C} 50$ hours after egg laying

470 (70 hours before the third instar stage) as before and then dissected.

471 Negative labeling of s/p loss of function clones- UbiFRT4OARFP/CyO flies (BDSC 34500) were

472 crossed with flies of genotype ywhsFlp;Sp/CyO; u8772 220bp>GFP (enhancer1) and ywhsFlp

$473 ; S p / C y O ; d 5778$ 870bp>GFP (enhancer2) to create strains with genotypes ywhsFlp;

474 Ubi>RFPFRT40A; u8772 220bp>GFP and ywhsFlp; Ubi>RFPFRT40A; d5778 870bp>GFP

475 respectively. Flies of genotype ywhsFlp; SlpS37A/Sm6-Tm6B flies (a kind gift from Dr. Andrew 
476 Tomlinson) were then crossed to ywhsFlp; Ubi>RFPFRT40A; u8772 220bp>GFP and ywhsFlp;

477 Ubi>RFPFRT40A; $d 5778$ 870bp>GFP and larvae were heat shocked for 45 minutes 48 hours after

478 egg laying. Third instar larvae were then dissected. The ywhsFlp; SIpS37A/Sm6-Tm6B strain

479 carries a deletion on chromosome II that spans both SIp1 and SIp2 genes. Clones carrying two

480 copies of this deficiency are seen as dark regions amidst Ubi>RFP marked wild type neuroblasts.

\section{Bioinformatic identification of TFBS}

482 Initially, to identify a list of possible candidate TFs with binding sites within our enhancers we 483 analyzed our enhancer sequences using the MEME suite tools ${ }^{78}$ and TOMTOM ${ }^{79}$ and the also by 484 an analytic tool developed at EPFL (https://biss.epfl.ch) that uses FIMO ${ }^{80}$ to identify motifs on a 485 sequence (Michael Frochaux, Dr.Bart Deplancke personal communication) to complement our 486 findings from TOMTOM. However mutating sites identified by this initial round of analysis did not always result in GFP reporter expression patterns that were consistent with our observations from the genetic experiments (data not shown). We suspected this could be because these analyses may have not exhaustively identified all potential binding sites for our regulators. To this end we employed a slightly different strategy to identify all possible binding sites of Ey, Su(H), and Scro whose knockdown had affected the expression of GFP reporters in

492 genetic experiments. We searched for consensus motifs of transcription factor binding sites for Ey, Su(H), Slp1 in JASPAR ${ }^{81,82}$ and Fly Factor Survey ${ }^{83}$ and then searched for potential matches to

494 these consensus sequences in our enhancers using the DNA Pattern Recognition program in 495 Sequence Manipulation Too ${ }^{65}$. Since consensus motifs for Scro binding sites were not 496 annotated in either JASPAR or Fly Factor Survey we instead picked 20 related transcription 497 factors of the NK-domain TF family whose consensus binding motifs were annotated (listed in 
Supplementary Table 2). We looked for potential matches to these motifs within our enhancers using the Pattern Recognition Tool of the Sequence Manipulation Suite. Sequences that were most shared between the 20 NK domain containing TFs we examined were scored as the most

501 likely motifs for Scro binding in the d5778 870bp enhancer. Given the smaller size of the 48772 been suggested to share the greatest homology to vnd of all other NK domain containing TFs ${ }^{55}$.

\section{Plasmids constructs and making transgenic fly stocks}

Primer sequences for all cloning are provided in a supplementary table (Supplementary Table

507 For making all constructs DNA was amplified from template using Expand High-Fidelity

CA.

511 Generating constructs for reporter assays and enhancer bashing and making transgenic

512 reporter expressing stocks- Sequences corresponding to the fragments of regulatory DNA

513 encoded in GMR35H02 and the d5778 REDfly enhancer were cloned from the BAC clone CH321-

$51494018^{84}$ (BACPAC resources). Sequences were PCR amplified from this BAC and cloned into

515 pJR12 vector (a kind gift from Dr. Jens Rister) between Ascl and Not/ sites. Transgenes were

516 inserted at the landing site VK00027 on the third chromosome (BL9744) by $\phi C 31$ integrase

517 mediated transgenesis ${ }^{85}$ and positive transformants were screened using the $w+$ marker

518 originally present in the pJR12 plasmid. Mutated enhancers were custom synthesized as gene 
blocks (gBlocks,IDT DNA) and cloned as stated above. Split gBlocks were custom made for making the $\mathrm{d} 5778$ ey site mutant and the $\mathrm{d} 5778 \mathrm{su}(\mathrm{h})$ site mutants. These split gBlocks were then PCR spliced and cloned between Notl and Ascl sites of the pJR12 vector. A custom-made

522 gBlock was used for making the d5778 scro mutant reporter. All reporters were integrated at

523 the same genomic site to ensure comparability across constructs and experiments.

524 Generation of CRISPR enhancer deletion constructs and transgenic stocks- CRISPR gRNAs were

525 designed by entering sequences of genomic DNA of the target +/- 20 kbp into the CRISPR

526 Optimal Target Finder web utility ${ }^{86}$ (https://www.targtfinder.flycrispr.neuro.brown.edu). Four

527 gRNAs-two upstream and two downstream of the target region to be deleted were then

528 selected. All four gRNAs were then cloned into the vector pCFD5 ${ }^{87}$ (Addgene plasmid \#73914 a

529 kind gift from Dr. Simon Bullock) using NEB-Builder HiFi DNA Assembly master mix. Constructs

530 were then injected into fly strain containing $M\{$ nos-cas9\}ZH-2A (BL54591) along with a $120 \mathrm{bp}$

531 repair oligonucleotide that contained 60bp of wild type genome sequence flanking both ends of

532 the expected double-stranded break. After injection, the nos-cas9 source was eliminated in the

533 subsequent generations by crossing individual G0 progeny flies to a double balancer of

534 genotype ywhsFlp; $\mathrm{Sp} / \mathrm{CyO}$; Tm2/Tm6B and selecting male G1 progeny. Individual G1 males

535 were then crossed to the same double balancer line to create stocks. The G1 males were then

536 genotyped by PCR to identify whether their genome had been edited, using RedExtract-n-Amp

537 tissue PCR kit (Sigma-Aldrich). G2 progeny of genome-edited males were then raised until

538 homozygous stocks were established.

539 Generation of the heat shock inducible NICD construct: We modified the pJR12 vector used in

540 enhancer bashing experiments to replace the GFP coding sequence with coding sequences of 
541 our interest. First, we generated a DNA fragment that matched the sequences of the pJR12

542 vector flanking the eGFP sequence but that did not contain the eGFP sequence itself by PCR

543 splicing. We then cut-out the eGFP segment from the pJR12 vector and replaced it with our GFP

544 deleted fragment using Nsil and Xhol. Next, an FRT-stop-FRT3-FRT segment was cloned from

545 the CoinFlp plasmid ${ }^{88}$ (Addgene plasmid \# 52889, a kind gift from Dr. Iswar Hariharan) and PCR

546 spliced with a fragment of Notch Intracellular Domain (NICD). Sequences corresponding to the

547 Notch Intracellular Domain were PCR amplified from Notch cDNA LD34134 (DGRC). The FRT-

548 stop-FRT3-FRT-NICD fragment was then cloned into the modified $\mathrm{pJR} 12$ vector between the

549 Pmel and Agel restriction sites. The deadpan enhancer sequence amplified from the BAC clone

$550 \mathrm{CH} 321-86 \mathrm{~A} 18$ (BACPAC resources) was then cloned into the modified $\mathrm{pJR} 12$ vector to drive the

551 heat shock inducible NICD expressing construct in presence of a heat shock activated Flippase

552 or hsFlp (this construct is abbreviated as $d p n>h s N I C D$ ). Construct was inserted at the VK00027

553 landing site (BL 9744) by $\phi C 31$ integrase mediated transgenesis. Positive transformants were

554 identified by the $\mathrm{w}+$ eye color marker expression and stocks were created by crossing to a

555 double balancer of genotype ywhsFlp; Sp/CyO; Tm2/Tm6B.

556 Generation of DamID-fusion constructs (UAS Dam-Ey and UAS-Dam-Su(H)) and transgene

557 expressing stocks- Su(H) coding sequence was amplified from a cDNA clone GH10914 (DGRC) by

558 PCR. The amplified Su(H) coding sequence was cloned into the pUAST-Dam-attB vector ${ }^{67}$ (a kind

559 gift from Dr. Andrea Brand) between the Notl and Xhol sites. A gBlock of Ey coding sequence

560 was ordered from IDT and cloned into the pUAST-Dam-attB vector. Constructs were then

561 incorporated at the VK00027 landing site (BL 9744) by $\phi C 31$ mediated integration. 
562 Transformants were identified by expression of the w+ marker gene. Final stocks were established by crossing to a double balancer line of genotype ywhsFlp; Sp/CyO; Tm2/Tm6B.

564 Immunofluorescence staining

565 Antibody staining was carried out as described in ${ }^{19}$ with a few modifications. The protocol is 566 described briefly as follows: brains from climbing third instar larvae were dissected in 1XPBS

567 and fixed in 4\% Formaldehyde solution in 1X PBS for 30 minutes on ice. Brains were then

568 incubated in primary antibody solution overnight at $4^{\circ} \mathrm{C}$, washed three times for 30 minutes

569 each at $4^{\circ} \mathrm{C}$, then incubated in fluor conjugated- secondary antibody solution overnight at $4^{\circ} \mathrm{C}$

570 and then washed again thrice at room temperature each time for 30 minutes. Samples were

571 mounted in Slowfade Gold antifade reagent (Invitrogen). Images are acquired using a Zeiss

572 LSM500 Confocal Microscope.

573 Antibodies used in the study are as follows: rabbit anti-SIp1, guinea-pig anti-SIp2, rabbit anti-Ey

574 (all used at 1:500) were kind gifts from Dr. Claude Desplan, guinea-pig anti-Dpn (1:500) (a kind

575 gift from Dr. Chris Doe). Commercially available antibodies include - sheep anti-GFP (1:500,

576 AbD Serotec, 4745-1051), Chicken anti-beta-gal (1:500, Abcam ab9361), Rat anti-Deadpan

577 [11D1BC7] (1:200, Abcam, ab195173) mouse anti-PCNA (1:50, Abcam ab29). These antibodies

578 are provided by the Developmental Studies Hybridoma Bank (DSHB): mouse anti-eyeless (1:10),

579 mouse anti-Pros (MR1A 1:20). Secondary antibodies are from Jackson or Invitrogen.

$580 \quad$ Fluorescence In situ hybridization (FISH)

581 FISH was carried out using custom made Stellaris probe sets as described in ${ }^{89}$ except that

582 incubation with sodium borohydride was skipped. Probes were generated using the slp1 mRNA 
and slp2 mRNA as templates. Concurrent FISH and immunofluorescence staining was carried out as described in the same publication.

\section{DamID-Seq}

ywhsFlp; Sp/CyO; UAS-Su(H)-Dam /Tm6B were crossed with Dcr2; tubG80ts; SoxNGal4. Mating

larval brains by shifting the larvae to $29^{\circ} \mathrm{C}$ for 72 hours. Climbing third instar larvae were then

around 100 brains were dissected.

Dam-ID libraries were made as described in ${ }^{90}$. Briefly, fly brains were dissected in 1X PBS and

602 then further enriched by PCR amplification, checked for quality and fragment size distribution 
604 Functional Genomics Unit of Roy J. Carver Biotechnology Center, UIUC). Libraries that were

605 deemed acceptable were then sequenced on a single SP100 lane of an Illumina NovaSeq

606

sequencer. Read lengths were 100bp. Two Biological replicates each of Ey-Dam, Su(H)-Dam and

607 Dam-only samples were sequenced, and each biological replicate is defined as one library

608

generated from $\sim 100$ dissected brains. Number of reads obtained were as follows: Dam

609

replicate1: 53745480 reads, Dam replicate 2: 85637355 reads, Ey-Dam replicate 1: 81121107

610

reads, Ey-Dam replicate 2: 82085708 reads, SuH-Dam replicate 1: 77448608 reads, replicate 2:

80015207 reads. All samples exhibited good QC scores.

\section{DamID-Seq data analysis}

613 DamID-seq data was analyzed using the damidseq-pipeline ${ }^{91}$. Duplicate samples of Ey-Dam and

$614 \mathrm{Su}(\mathrm{H})$-Dam samples and Dam-only controls were aligned to the Drosophila reference genome

615 UCSC dm6. Alignment rate for individual samples were as follows:(Ey-Dam replicate 1- 97.04\%,

616 Ey-Dam replicate 2 -97.82\%, Su(H)-Dam replicate 1- 97.91\% and Su(H)-Dam replicate 2 -

$61797.82 \%$. The main utility- the damid-seqpipeline was used to align reads to the genome using

618 bowtie2, bin and count reads, normalize counts and generate log-2 ratio bedgraph files for each

619 DamID-sample and its corresponding Dam-only control. The provided gatc.track file was used

620 for running the script. Next, the findpeaks utility was used with an F.D.R. $<0.01$ to identify

621 peaks. We then used the provided peaks2genes utility to assign peaks to genes. To assess the

622 reproducibility of our data we also ran the findpeaks script using F.D.R $<0.1$ to discover peaks

623 with weaker statistical confidence and used this as input for the I.D.R. Python package

624 (https://github.com/nboley/idr). 984 of 1810 (54.4.\%) Ey-Dam and 972 of 1996 (48.7\%) of

$625 \mathrm{Su}(\mathrm{H})$-Dam peaks passed an I.D.R. threshold of 0.05. Lists of genes bound reproducibly by both 
626 Ey-Dam and by Su(H)-Dam were used in BioVenn ${ }^{92}$ to visualize the overlap between these two

627 lists and estimate percentage of genes regulated by both transcription factors. Enriched gene

628 ontology terms in this list were identified using DAVID ${ }^{93}, 94$. All replicate tracks for Ey and for

$629 \mathrm{Su}(\mathrm{H})$ as well as reproducible peaks identified using I.D.R. were visualized in IGV ${ }^{95}$.

$630 \quad$ Image quantification and statistical analysis.

631 No statistical method was used to predetermine sample size. Sample size /replicate numbers

632 were decided based on routine in the field and previous experience. All data were obtained

633 from at least two independent experiments. The numbers of animals or clones analyzed can be

634 found in figure legends or this section (see below). All data are highly reproducible. No outliers

635 are excluded. For specific quantification method and statistical analysis see below.

636 Imaging and image analysis for comparison of wild type and mutant GFP reporter intensities- All

637 images were acquired using a Zeiss LSM 500 confocal microscope. Samples were imaged at sub-

638 saturating illumination conditions, with stack intervals of 1 micron. Same laser settings and

639 imaging conditions were used across all variants of the same enhancers. Image analysis was

640 carried out using Fiji ${ }^{96}$. Signal intensities in the GFP and the Dpn channels were measured over

641 a small rectangular selection for all variants. For the same enhancer a 'scaling factor' was

642 calculated for each mutant by dividing the Dpn channel intensity for mutant reporter by the

643 channel intensity of the corresponding wild type reporter. The GFP channel intensities for each

644 variant was then multiplied by this scaling factor. Finally a ratio of the scaled GFP channel

645 intensity to the Dpn channel intensity was calculated for each GFP reporter variant. Ratios of

646 scaled GFP/Dpn intensities was then plotted using GraphPad Prism and for each enhancer RM 
647 One-way ANOVA with Geisser-Greenhouse correction and Tukey's multiple comparisons were

648 carried out between reporter variants of the same enhancer. For the $d 5778870$ bp enhancer

649 five sets of data from twenty different optic lobes were acquired for each enhancer (one set-

650 wild type and all mutant variations all imaged using the same conditions). For the $48772220 \mathrm{bp}$

651 enhancer six sets of data were used for quantification.

652 Image analysis for CRISPR enhancer deletion experiments- All images were acquired using a

653 Zeiss LSM 500 confocal microscope under sub-saturating illumination conditions. For each

654 sample width of SIp1, SIp2 and Dpn expression domains was measured in Fiji at five different

655 locations and averaged. The brain size of the sample was estimated by measuring the length of

656 the brain at its widest point perpendicular to the neuroblast division axis. The width of each

657 protein expression domain was divided by the brain size to normalize expression domain size

658 estimates to the brain size. For each protein SIp1, SIp2 and Dpn- these ratios were then plotted

659 in GraphPad Prism and ordinary one way ANOVA and Dunnett's multiple comparisons tests

660 were carried out between wild type and CRISPR enhancer deleted brains. Data from seven

661 brains of each genotype was analyzed and quantified.

662 All images were processed in Adobe Photoshop and assembled using Adobe Illustrator.

663 Data Availability

664 The raw and processed DamID-seq data have been deposited to GEO, and the accession 665 number is GSE188643 (reviewer's token qnonwyyidtsndcp). 
667 We thank the Functional Genomics Unit and the DNA-Sequencing Services Unit of the Roy J.

668 Carver Biotechnology Center at the University of Illinois at Urbana-Champaign for assistance with

669 quality control and sequencing of Dam-ID sequencing libraries. We thank the fly community,

670 especially Claude Desplan, Andrea Brand, Chris Doe, Bruce Edgar, Kenneth Irvine, Miki Fujioka

671 and James B. Jaynes for generous gifts of antibodies and fly stocks. We are grateful to Bart

672 Deplancke' team for help with the YIH pipeline to predict transcription factor binding sites within

673 enhancers. We thank the Bloomington Drosophila Stock Center, the Vienna Drosophila RNAi

674 Center, the Developmental Studies Hybridoma Bank, and TriP at Harvard Medical School

675 (NIH/NIGMS R01-GM084947) for fly stocks and reagents. We thank members of the Li lab Dr. Yu

676 Zhang and Hailun Zhu for helpful discussions. This work was supported by the National Eye

677 Institute (Grant 1 R01 EY026965-01A1) and start-up funds from the University of Illinois at

678 Urbana-Champaign.

679 Competing interests.

680 The authors declare no competing or financial interests.

681 Materials \& Correspondence. Publicly available fly lines (those from BDSC or VDRC) should be

682 requested directly from the corresponding stock centers: https://bdsc.indiana.edu/ or

683 https://stockcenter.vdrc.at/control/main. Fly lines generated in this study can be requested

684 without restriction. Correspondence and requests for materials should be addressed to Xin $\mathrm{Li}$

685 (lixin@illinois.edu).

686 References

6871 Lin, S. \& Lee, T. Generating neuronal diversity in the Drosophila central nervous system. Dev Dyn

$688 \quad 241,57-68$, doi:10.1002/dvdy.22739 (2012). 
689

690

691

692

693

694

695

696

697

698

699

700

701

702

703

704

705

706

707

708

709

710

711

712

713

714

715

716

717

718

719

720

721

722

723

724

725

726

727

728

729

730

731

732

733

734

735

2 Allan, D. W. \& Thor, S. Transcriptional selectors, masters, and combinatorial codes: regulatory principles of neural subtype specification. Wiley Interdiscip Rev Dev Biol 4, 505-528, doi:10.1002/wdev.191 (2015).

3 Holguera, I. \& Desplan, C. Neuronal specification in space and time. Science 362, 176-180, doi:10.1126/science.aas9435 (2018).

4 Sagner, A. \& Briscoe, J. Establishing neuronal diversity in the spinal cord: a time and a place. Development 146, doi:10.1242/dev.182154 (2019).

5 Pearson, B. J. \& Doe, C. Q. Specification of temporal identity in the developing nervous system. Annu Rev Cell Dev Biol 20, 619-647, doi:10.1146/annurev.cellbio.19.111301.115142 (2004).

6 Oberst, P., Agirman, G. \& Jabaudon, D. Principles of progenitor temporal patterning in the developing invertebrate and vertebrate nervous system. Curr Opin Neurobiol 56, 185-193, doi:10.1016/j.conb.2019.03.004 (2019).

7 Maurange, C. Temporal patterning in neural progenitors: from Drosophila development to childhood cancers. Dis Model Mech 13, doi:10.1242/dmm.044883 (2020).

8 Doe, C. Q. Temporal Patterning in the Drosophila CNS. Annu Rev Cell Dev Biol 33, 219-240, doi:10.1146/annurev-cellbio-111315-125210 (2017).

9 Miyares, R. L. \& Lee, T. Temporal control of Drosophila central nervous system development. Curr Opin Neurobiol 56, 24-32, doi:10.1016/j.conb.2018.10.016 (2019).

10 Brody, T. \& Odenwald, W. F. Programmed transformations in neuroblast gene expression during Drosophila CNS lineage development. Dev Biol 226, 34-44, doi:10.1006/dbio.2000.9829 (2000).

11 Isshiki, T., Pearson, B., Holbrook, S. and Doe, C.Q. . Drosophila neuroblasts sequentially express transcription factors which specify the tmporal identity of their neuronal progeny Cell 106, 511521 (2001).

12 Grosskortenhaus, R., Robinson, K. J. \& Doe, C. Q. Pdm and Castor specify late-born motor neuron identity in the NB7-1 lineage. Genes Dev 20, 2618-2627, doi:10.1101/gad.1445306 (2006).

13 Baumgardt, M., Karlsson, D., Terriente, J., Diaz-Benjumea, F. J. \& Thor, S. Neuronal subtype specification within a lineage by opposing temporal feed-forward loops. Cell 139, 969-982, doi:10.1016/j.cell.2009.10.032 (2009).

$14 \mathrm{Li}, \mathrm{X}$., Chen, Z. \& Desplan, C. Temporal patterning of neural progenitors in Drosophila. Curr Top Dev Biol 105, 69-96, doi:10.1016/B978-0-12-396968-2.00003-8 (2013).

15 Suzuki, T., Kaido, M., Takayama, R. \& Sato, M. A temporal mechanism that produces neuronal diversity in the Drosophila visual center. Dev Biol 380, 12-24, doi:10.1016/j.ydbio.2013.05.002 (2013).

16 Konstantinides, N. et al., A comprehensive series of temporal transcription factors in the fly visual system. Biorxiv (as accessed on 10/28/2021) doi:10.1101/2021.06.13.448242 (2021).

17 Zhu, H., Zhao, S. D., Ray, A., Zhang, Y. \& Li, X., A comprehensive temporal patterning gene network in Drosophila medulla neuroblasts revealed by single-cell RNA sequencing. Biorxiv (as accessed on 10/28/2021) doi:10.1101/2021.06.12.448145 (2021).

18 Tang, J. L. Y. et al., NanoDam identifies novel temporal transcription factors conserved between the Drosophila central brain and visual system. Biorxiv (as accessed on 10/28/2021) doi:10.1101/2021.06.07.447332 (2021).

$19 \mathrm{Li}$, X. et al. Temporal patterning of Drosophila medulla neuroblasts controls neural fates. Nature 498, 456-462, doi:10.1038/nature12319 (2013).

20 Maurange, C., Cheng, L. \& Gould, A. P. Temporal transcription factors and their targets schedule the end of neural proliferation in Drosophila. Cell 133, 891-902, doi:10.1016/j.cell.2008.03.034 (2008). 
21 Averbukh, I., Lai, S. L., Doe, C. Q. \& Barkai, N. A repressor-decay timer for robust temporal patterning in embryonic Drosophila neuroblast lineages. Elife 7, doi:10.7554/eLife.38631 (2018).

22 Hirono, K., Margolis, J. S., Posakony, J. W. \& Doe, C. Q. Identification of hunchback cis-regulatory DNA conferring temporal expression in neuroblasts and neurons. Gene Expr Patterns 12, 11-17, doi:10.1016/j.gep.2011.10.001 (2012).

23 Grosskortenhaus, R., Pearson, B. J., Marusich, A. \& Doe, C. Q. Regulation of temporal identity transitions in Drosophila neuroblasts. Dev Cell 8, 193-202, doi:10.1016/j.devcel.2004.11.019 (2005).

24 Mettler, U., Vogler, G. \& Urban, J. Timing of identity: spatiotemporal regulation of hunchback in neuroblast lineages of Drosophila by Seven-up and Prospero. Development 133, 429-437, doi:10.1242/dev.02229 (2006).

25 van den Ameele, J. \& Brand, A. H. Neural stem cell temporal patterning and brain tumour growth rely on oxidative phosphorylation. Elife 8, doi:10.7554/eLife.47887 (2019).

26 Okamoto, M. et al. Cell-cycle-independent transitions in temporal identity of mammalian neural progenitor cells. Nat Commun 7, 11349, doi:10.1038/ncomms11349 (2016).

27 Moore, R. \& Alexandre, P. Delta-Notch Signaling: The Long and The Short of a Neuron's Influence on Progenitor Fates. J Dev Biol 8, doi:10.3390/jdb8020008 (2020).

28 Fortini, M. E. Notch signaling: the core pathway and its posttranslational regulation. Dev Cell 16, 633-647, doi:10.1016/j.devcel.2009.03.010 (2009).

29 Kopan, R. \& Ilagan, M. X. The canonical Notch signaling pathway: unfolding the activation mechanism. Cell 137, 216-233, doi:10.1016/j.cell.2009.03.045 (2009).

30 Bray, S. \& Bernard, F. in Notch Signaling Current Topics in Developmental Biology 253-275 (2010).

31 Walsh, K. T. \& Doe, C. Q. Drosophila embryonic type II neuroblasts: origin, temporal patterning, and contribution to the adult central complex. Development 144, 4552-4562, doi:10.1242/dev.157826 (2017).

32 Knoblich, J. A., Jan Lily Y., and Jan Yuh Nung. Asymmetric segregation of Numb and Prospero during cell division Nature 377, 624-627 (1995).

33 Rhyu, M., S. , Jan, Lily, Y., and Jan Yuh Nung Asymmetric segregation of Numb protein during division of the sensory organ precursor cell confers distinct fates to daughter cells. Cell 76, 477491 (1994).

34 Roegiers, F. \& Jan, Y. N. Asymmetric cell division. Curr Opin Cell Biol 16, 195-205, doi:10.1016/j.ceb.2004.02.010 (2004).

35 Fuerstenberg, S., Broadus, Julie, and Doe Chris, Q Asymmetry and cell fate in the Drosophila embryonic CNS. The International Journal of Developmental Biology 42, 379-383 (1998).

36 Wang, H. et al. Aurora-A acts as a tumor suppressor and regulates self-renewal of Drosophila neuroblasts. Genes Dev 20, 3453-3463, doi:10.1101/gad.1487506 (2006).

37 Bowman, S. K. et al. The tumor suppressors Brat and Numb regulate transit-amplifying neuroblast lineages in Drosophila. Dev Cell 14, 535-546, doi:10.1016/j.devcel.2008.03.004 (2008).

38 Weng, M., Golden, K. L. \& Lee, C. Y. dFezf/Earmuff maintains the restricted developmental potential of intermediate neural progenitors in Drosophila. Dev Cell 18, 126-135, doi:10.1016/j.devcel.2009.12.007 (2010).

39 Zacharioudaki, E., Magadi, S. S. \& Delidakis, C. bHLH-O proteins are crucial for Drosophila neuroblast self-renewal and mediate Notch-induced overproliferation. Development 139, 12581269, doi:10.1242/dev.071779 (2012).

40 Almeida, M. S. \& Bray, S. J. Regulation of post-embryonic neuroblasts by Drosophila Grainyhead. Mech Dev 122, 1282-1293, doi:10.1016/j.mod.2005.08.004 (2005). 
$784 \quad 41 \quad$ Magadi, S. S. et al., doi:10.1101/2020.03.25.007187 (2020).

$78542 \quad$ Ulvklo, C. et al. Control of neuronal cell fate and number by integration of distinct daughter cell proliferation modes with temporal progression. Development 139, 678-689, doi:10.1242/dev.074500 (2012).

$78843 \quad$ Bivik, C. et al. Control of Neural Daughter Cell Proliferation by Multi-level Notch/Su(H)/E(spl)-

789 HLH Signaling. PLoS Genet 12, e1005984, doi:10.1371/journal.pgen.1005984 (2016).

790 Kageyama, R., Ohtsuka, T., Shimojo, H. \& Imayoshi, I. Dynamic regulation of Notch signaling in neural progenitor cells. Curr Opin Cell Biol 21, 733-740, doi:10.1016/j.ceb.2009.08.009 (2009). Pfeiffer, B. D., Jenett, A., Hammonds, A.S., Ngo, T. B., Misra, S., Murphy, C., Scully, A., Carlson, J. W., Wan, K.H., Laverty, T.R., Myngall, C., Svirkas, R., Kadonaga, J.T., Doe, C.Q., Eisen, M.B., Celniker, S.E., and Rubin, G.M. . Tools for neuroanatomy and neurogenetics in Drosophila Proc Natl Acad Sci U S A 105, 9715-9720 (2008).

797 Jenett, A. et al. A GAL4-driver line resource for Drosophila neurobiology. Cell Rep 2, 991-1001, doi:10.1016/j.celrep.2012.09.011 (2012).

47 Li, H. H. et al. A GAL4 driver resource for developmental and behavioral studies on the larval CNS of Drosophila. Cell Rep 8, 897-908, doi:10.1016/j.celrep.2014.06.065 (2014). Single-base pair differences in a shared motif determine differential Rhodopsin expression. Science 350, 1258-1261 (2015). Frankel, N. et al. Phenotypic robustness conferred by apparently redundant transcriptional enhancers. Nature 466, 490-493, doi:10.1038/nature09158 (2010).

Fujioka, M. \& Jaynes, J. B. Regulation of a duplicated locus: Drosophila sloppy paired is replete with functionally overlapping enhancers. Dev Biol 362, 309-319, doi:10.1016/j.ydbio.2011.12.001 (2012). Ghiasvand, N. M. et al. Deletion of a remote enhancer near ATOH7 disrupts retinal neurogenesis, causing NCRNA disease. Nat Neurosci 14, 578-586, doi:10.1038/nn.2798 (2011). development. Trends Genet 28, 409-416, doi:10.1016/j.tig.2012.03.006 (2012). Gallo, S. M., Li, L., Hu, Z. \& Halfon, M. S. REDfly: a Regulatory Element Database for Drosophila. Bioinformatics 22, 381-383, doi:10.1093/bioinformatics/bti794 (2006).

54 Rivera, J., Keranen, S. V. E., Gallo, S. M. \& Halfon, M. S. REDfly: the transcriptional regulatory element database for Drosophila. Nucleic Acids Res 47, D828-D834, doi:10.1093/nar/gky957 (2019). pharynx, ventral nerve cord and brain of Drosophila embryos. Mechanisms of Development 94, 237-241 (2000).

830 Bailey, A. M. a. P., J. . Suppressor of Hairless directly activates transcription of enhancer of split complex genes in response to Notch receptor activity. Genes and Development 9, 2609-2622 (1995).

Furukawa, T., Kobayakawa, Y., Tamura, K., Kimura, K., Kawaichi, M., Tanimura, T., and Honjo, T. . Suppressor of Hairless, the Drosophila homolog of RBP-Jkappa, transactivates the neurogenic gene $\mathrm{E}(\mathrm{spl}) \mathrm{m8}$. Japan Journal of Genetics 70, 505-524 (1995).

58 Ito, K., Awano, W., Suzuki, K., Hiromi, Y., and Yamamoto, D. The Drosophila mushroom body is a quadruple structure of clonal units each of which contains a virtually identical set of neurones and glial cells Developemnt 124, 761-771 (1997).

59 Nusslein-Volhard, C., Wieschaus, E. and Kluding, H. . Mutations affecting the pattern of larval cuticle in Drosophila melanogaster I.Zygotic loci on the second chromosome Roux's archives of developmental biology 193, 267-282 (1984). 
A

Medulla neuroblasts

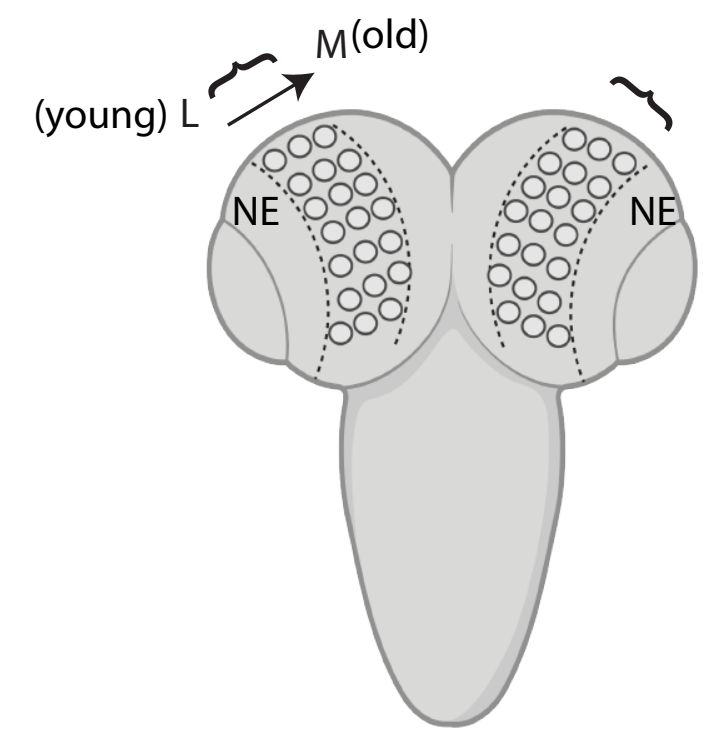

D

bioRxivprep R3: 5 H

(which was not cortified by /ai.org/10.1101/2021.10.29.466469; this version posted December 5, 2021. The copyright holder for this preprint

列

$\mathbf{u} 8772$

$\mathbf{d 5 7 7 8}$
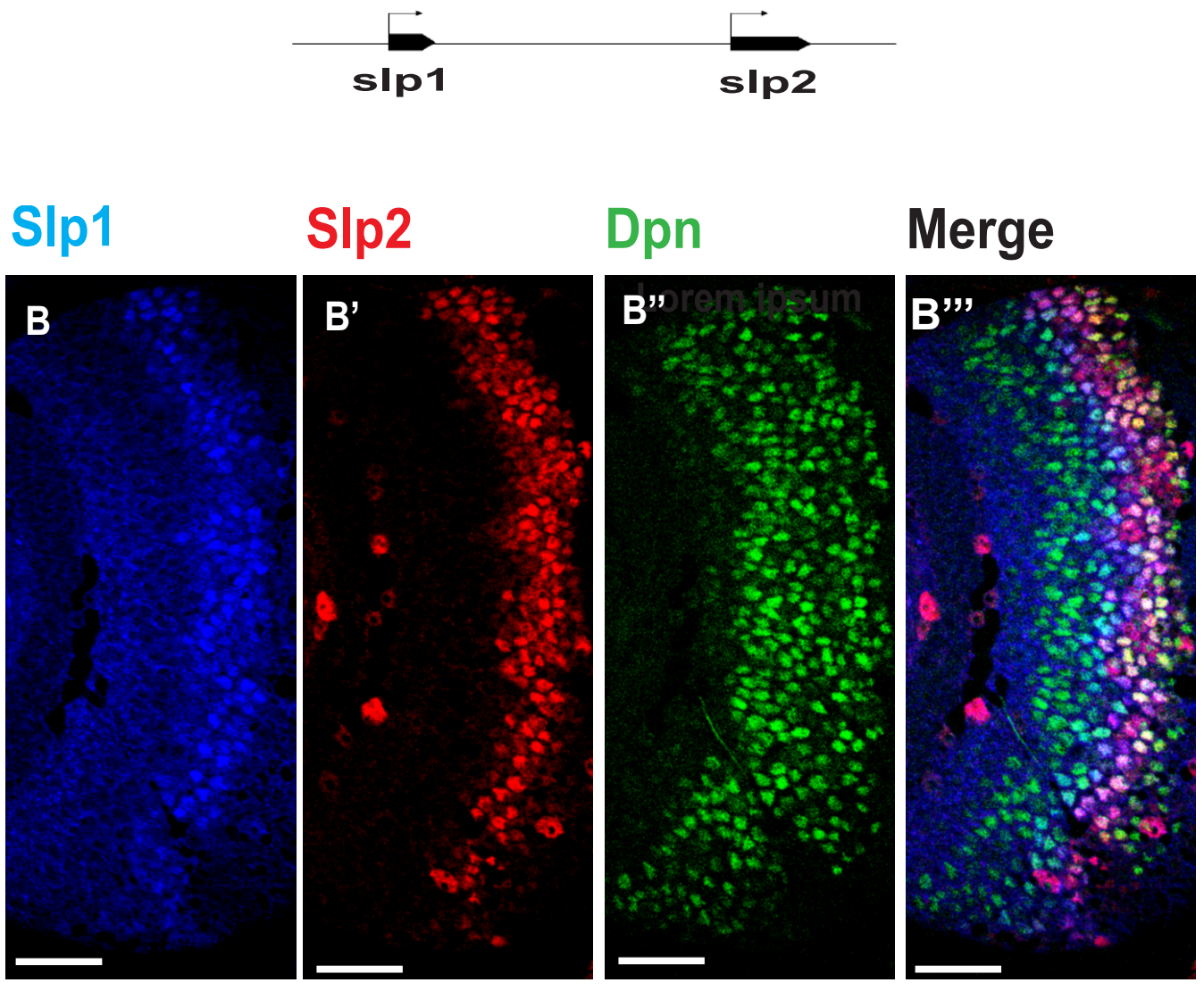

slp1 slp2

mRNA mRNA
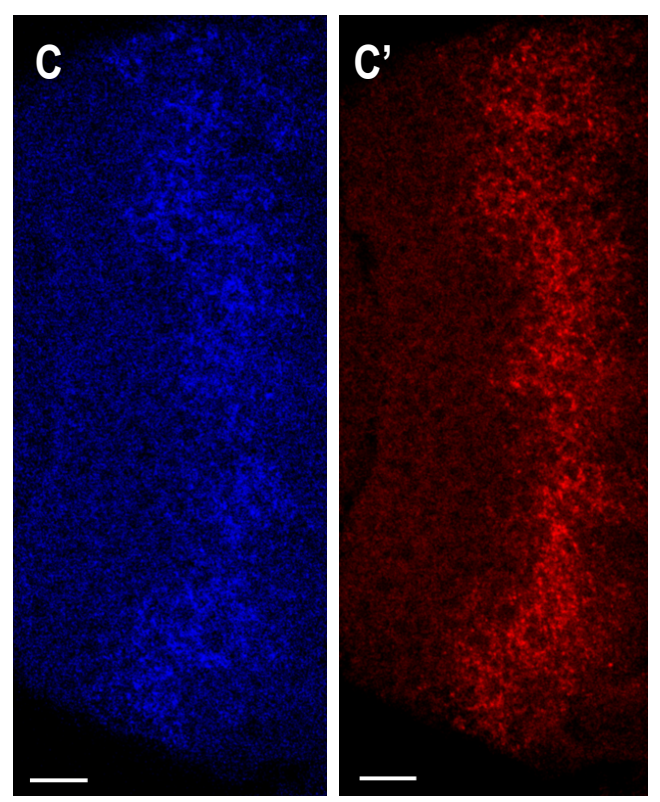

Dpn

Merge
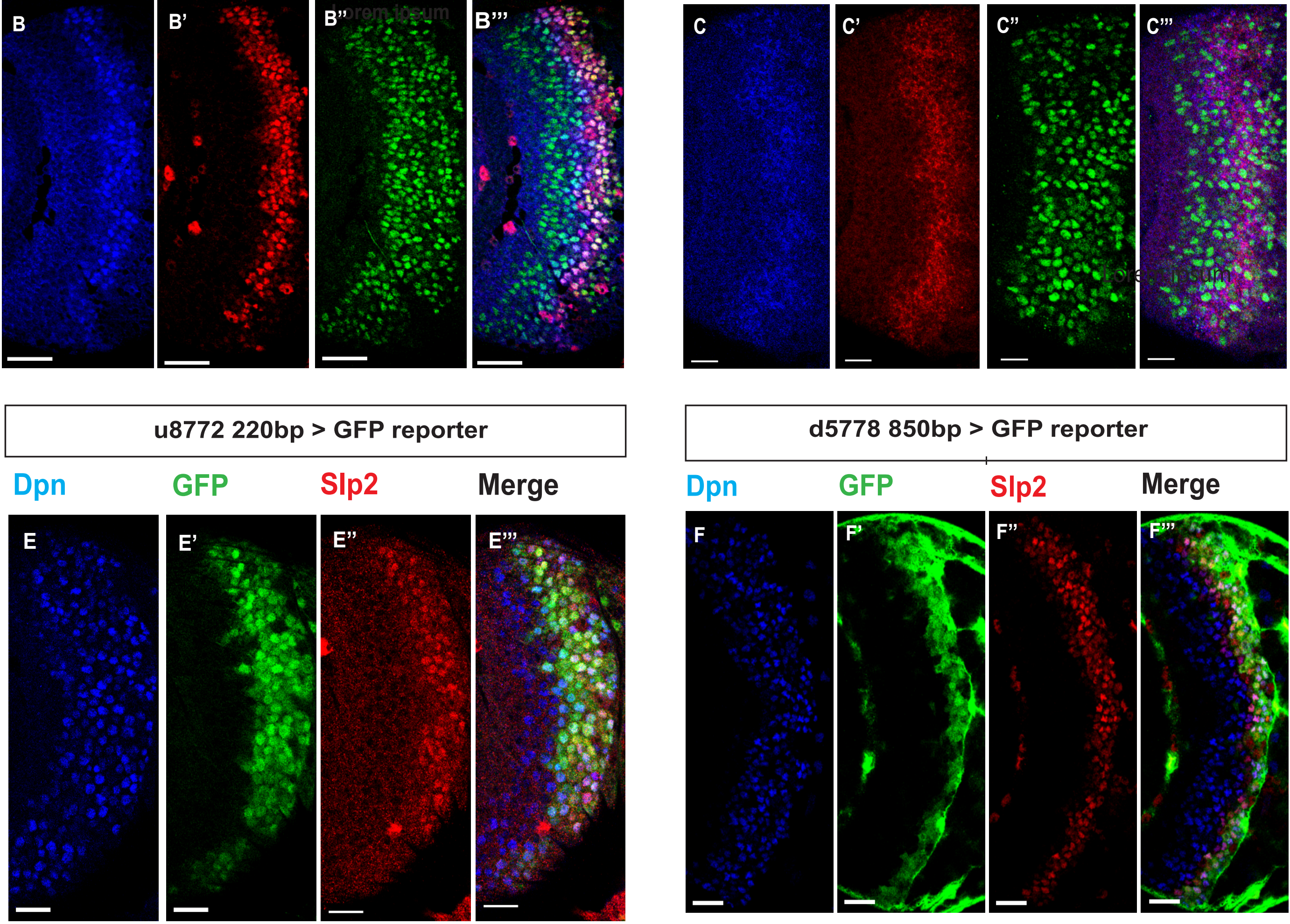

u8772 220bp > GFP reporter

\section{GFP SIp2 Merge}
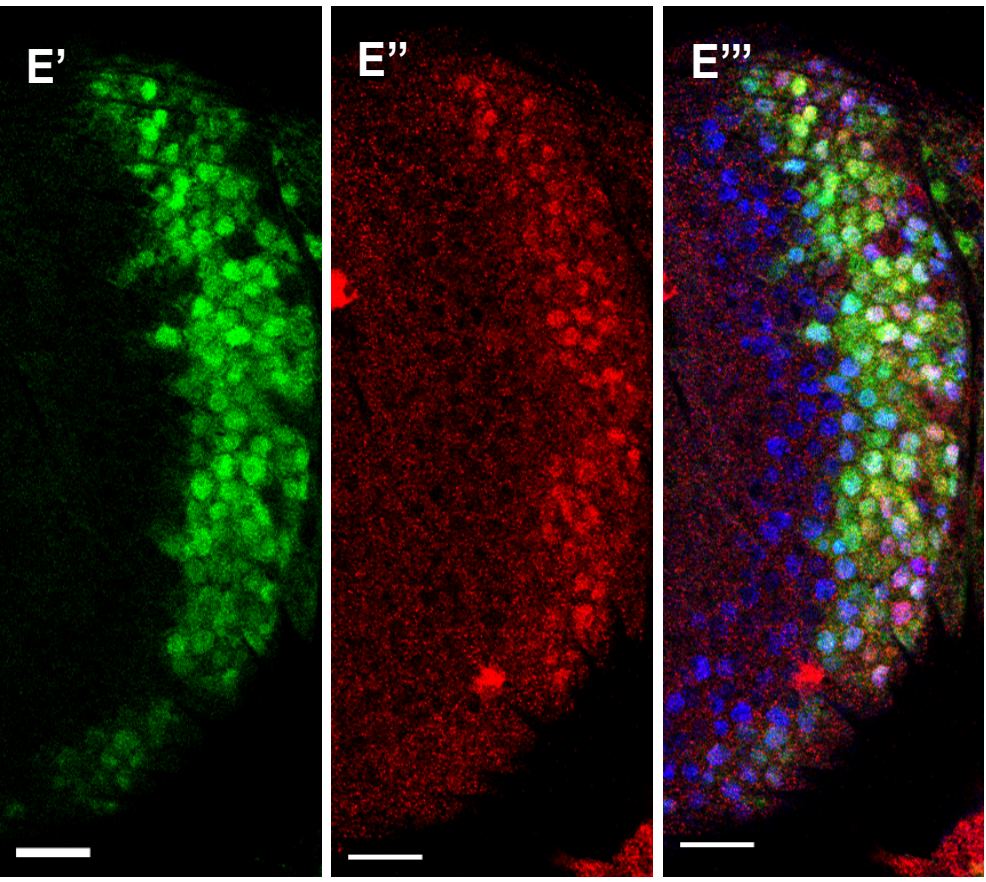

Old NBs
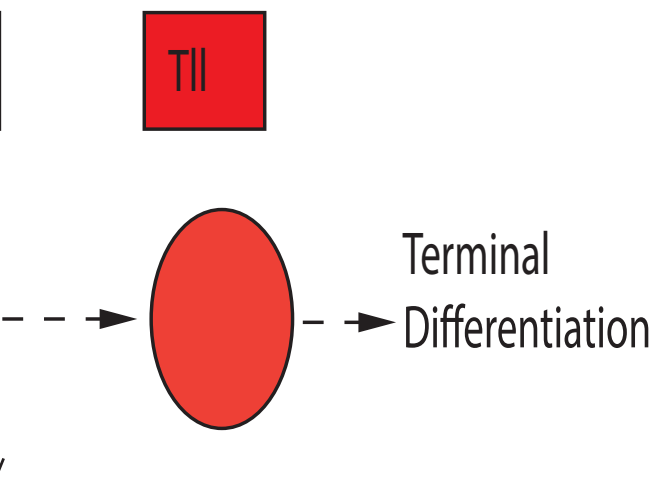
Figure 1. Expression of s/p1 and s/p2 genes in medulla neuroblasts is controlled at transcription and achieved by coordinated action of at least two transcriptional enhancers.

(A) Schematic of Drosophila brain at the third instar larval stage highlighting the location of the optic lobe medulla. Medulla NBs (shown as circles), located on the surface of the optic lobe, are transformed from neuroepithelial cells (NE) as a neurogenesis wave spreads in a medial to lateral direction. As a result, medulla NBs of different ages are aligned on the lateral $(L)$ to medial (M) spatial axis. Schematic to the right shows part of the temporal patterning program of medulla NBs with a focus on the Ey to SIp1/2 transition. NBs undergo asymmetric divisions to self-renew and to produce intermediate progenitors called Ganglion Mother Cells that each divide once to produce two neurons. In the Ey stage, NBs undergo a few divisions while gradually turning on SIp expression, thus there is a significant overlap between Ey and SIp expression in NBs. After Slp level reaches a certain threshold, the transition occurs. Several such transitions later as indicated by the dashed arrow neuroblasts express TTF Tailless before entering the final stage to undergo terminal differentiation. The larval brain graphic was created using BioRender. (B) Expression patterns of SIp1 and SIp2 proteins in medulla neuroblasts identified by their expression of Deadpan (Dpn, a neuroblast marker). (C) Expression patterns of SIp1 and SIp2 mRNAs in Dpn expressing medulla neuroblast closely parallels the corresponding protein expression patterns. (D) Schematic of the relative locations of s/p1 and s/p2 genes and transcriptional enhancers identified as regulating their expression. Positions of slp1 coding locus (2L:3,825,675..3,827,099 [+]), slp2 coding locus (2L:3,836,840..3,839,185 [+]), GMR35H02 (2L:3,817,265..3,821,014) and the REDfly enhancers u8772 (2L:3,816,967..3,818,532) and d5778 (2L:3,842,530..3,844,660) are shown relative to one another. A 220 bp enhancer located within the genomic segment covered by GMR35H02 and an 850 base pair enhancer element within the REDFly enhancer d5778 were identified as potential cis-regulatory elements of s/p1 and s/p2 genes. The distance between the start of GMR35H02 and the end of d5778 is around $27.394 \mathrm{kbp}$. (E) Expression patterns of a GFP reporter expressing GFP driven by the 220 bp enhancer closely approximates endogenous expressions of SIp1 and SIp2 in medulla neuroblasts. (F) Expression pattern of GFP reporter driven by the 850 bp enhancer shown alongside endogenous Slp1/2 expressions. This enhancer initiates GFP expression at a slightly later stage compared to the 220 bp enhancer and likely also regulates SIp2 expression in surface glia. Scale bar: 20 microns. 
Figure 2

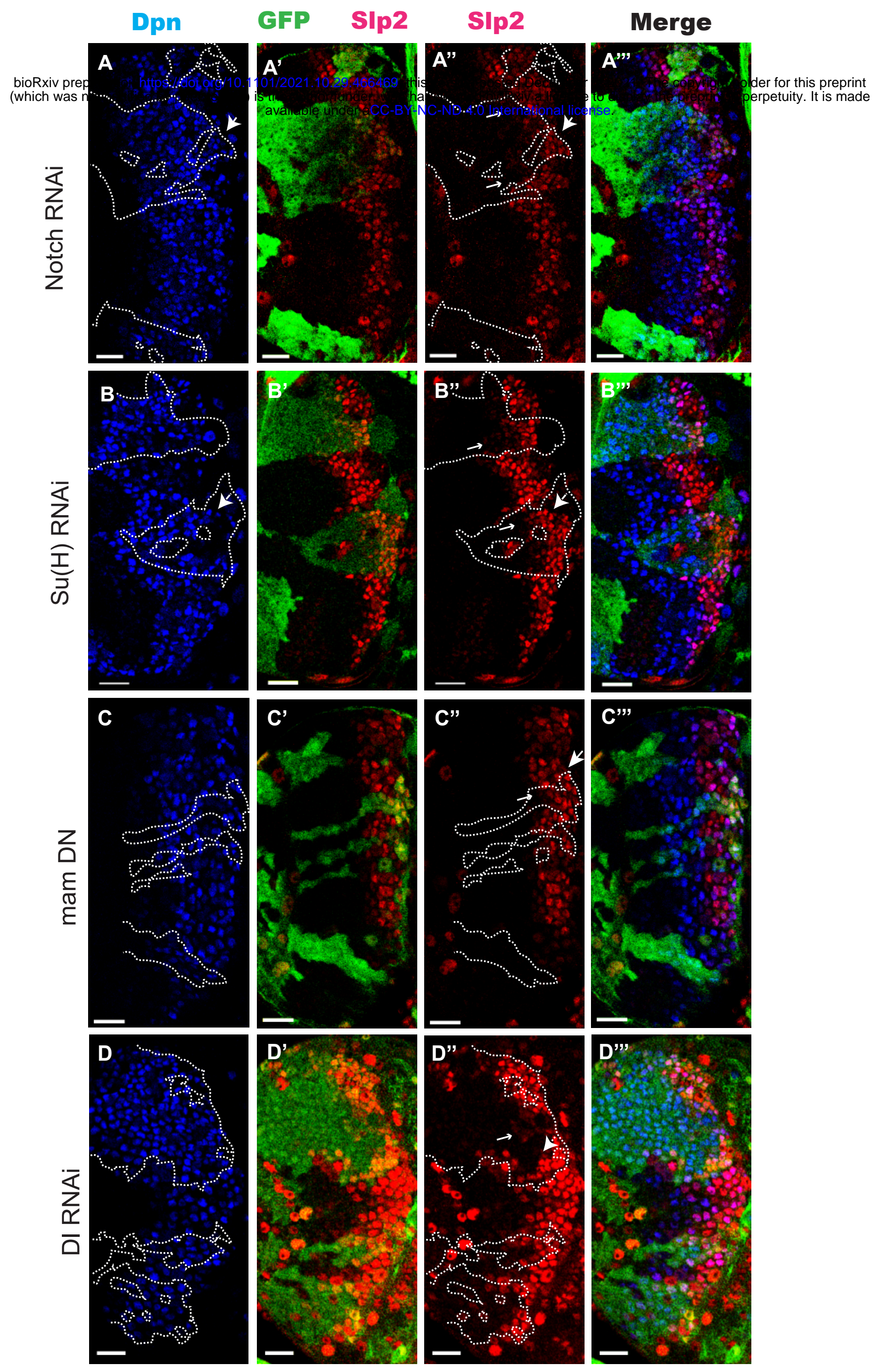


Figure 2. The Notch pathway regulates expression of slp genes in medulla neuroblasts. (A) RNAi knockdown of Notch in GFP marked clones leads to a delay in expression of SIp2 compared to contemporaneous wild type neuroblasts outside the GFP marked clones. (B) RNAi knockdown of $\mathrm{Su}(\mathrm{H})$ shows a similar delay in Slp2 expression as for Notch-RNAi. (C) Expression of a dominant negative variant of Mastermind, third component of the Notch transcription complex shows a similar phenotype as N RNAi and Su(H) RNAi and delays SIp2 expression. (D) Knockdown of DI, a Notch ligand, caused a similar delay in SIp2 expression. All clones are generated with ayGal4 UAS GFP and clone boundaries are shown in dotted lines. Arrows indicate that SIp2 expression is delayed in the clones. Scale bar 20 microns. 
Figure 3

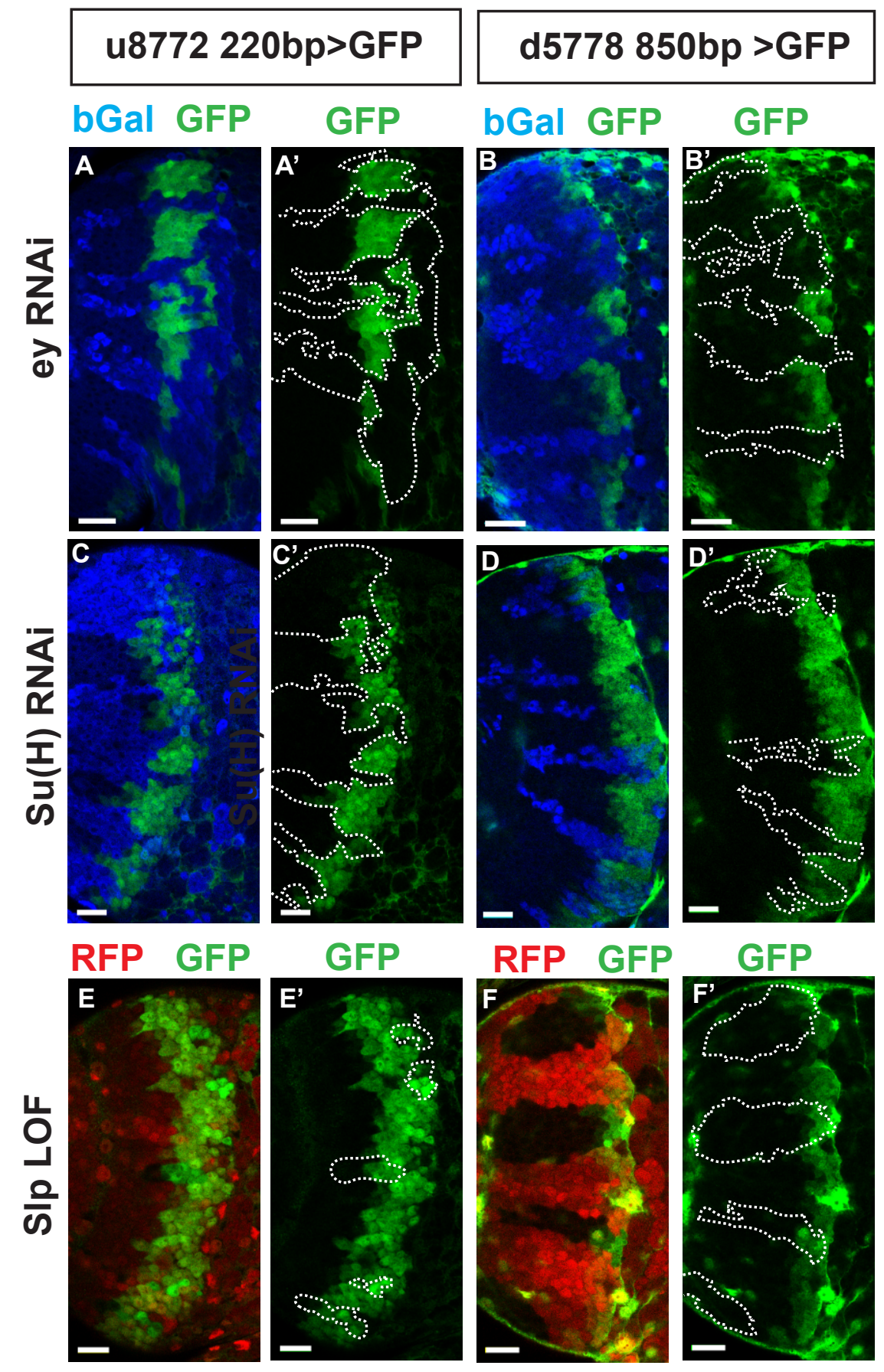


Figure 3. Eyeless and the Notch pathway act through the $\mathbf{2 2 0}$ bp and $\mathbf{8 5 0}$ bp enhancers to regulate expression of slp genes. (A-D') RNAi clones in which Ey or $\mathrm{Su}(\mathrm{H})$ is knocked down are marked by Beta-galactosidase $(b G a l)$ in blue, while regions unmarked by bGal are populated by wild type neuroblasts. ( $\left.A, A^{\prime}\right)$ Observed expression of a GFP reporter driven by the $220 \mathrm{bp}$ enhancer element in Ey RNAi clones and wild type neuroblasts. $\left(\mathrm{B}, \mathrm{B}^{\prime}\right)$ Observed expression of a GFP reporter driven by the 850 bp enhancer element in Ey RNAi knockdown clones and in wild type neuroblasts. In case of both enhancers knockdown of Ey leads to a loss of GFP reporter expression. $\left(C, C^{\prime}\right)$ The expression of the GFP reporter driven by $220 b p$ enhancer in $\mathrm{Su}(\mathrm{H}) \mathrm{RNAi}$ clones and in wild type neuroblasts. $\left(D, D^{\prime}\right)$ The expression of the GFP reporter driven by $850 b p$ enhancer in $\mathrm{Su}(\mathrm{H}) \mathrm{RNAi}$ clones and in wild type neuroblasts. In case of both enhancers expression of the GFP reporter is lost or delayed. (E, E') Expression of the 220 bp GFP reporter in s/p1 and s/p2 loss of function mitotic clones ('dark' regions) and in wild type neuroblasts (marked by RFP). (F,F') Expression of the 850 bp enhancer GFP reporter in s/p LOF clones and wild type neuroblasts similar to $\left(E, E^{\prime}\right)$. Slp1 binding sites were identified in both enhancer elements which indicate that SIp may auto-regulate their expression. However, GFP reporter expression driven by these two enhancers is not significantly affected within slp LOF neuroblasts, suggesting that SIp1/2 are not required to initiate their own expression in neuroblasts. Scale bar 20 microns. 
Figure 4

u8772 220 bp enhancer

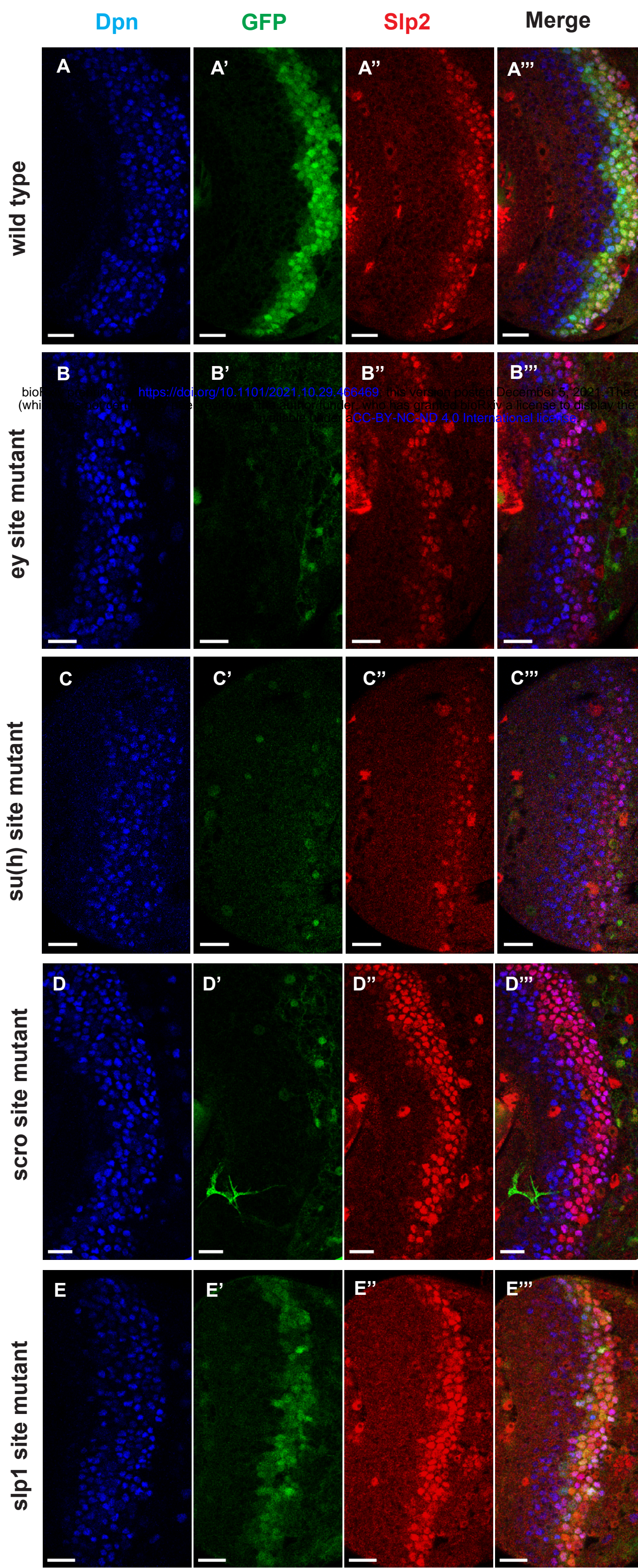

F $\quad$ u8772 220bp GFP reporters

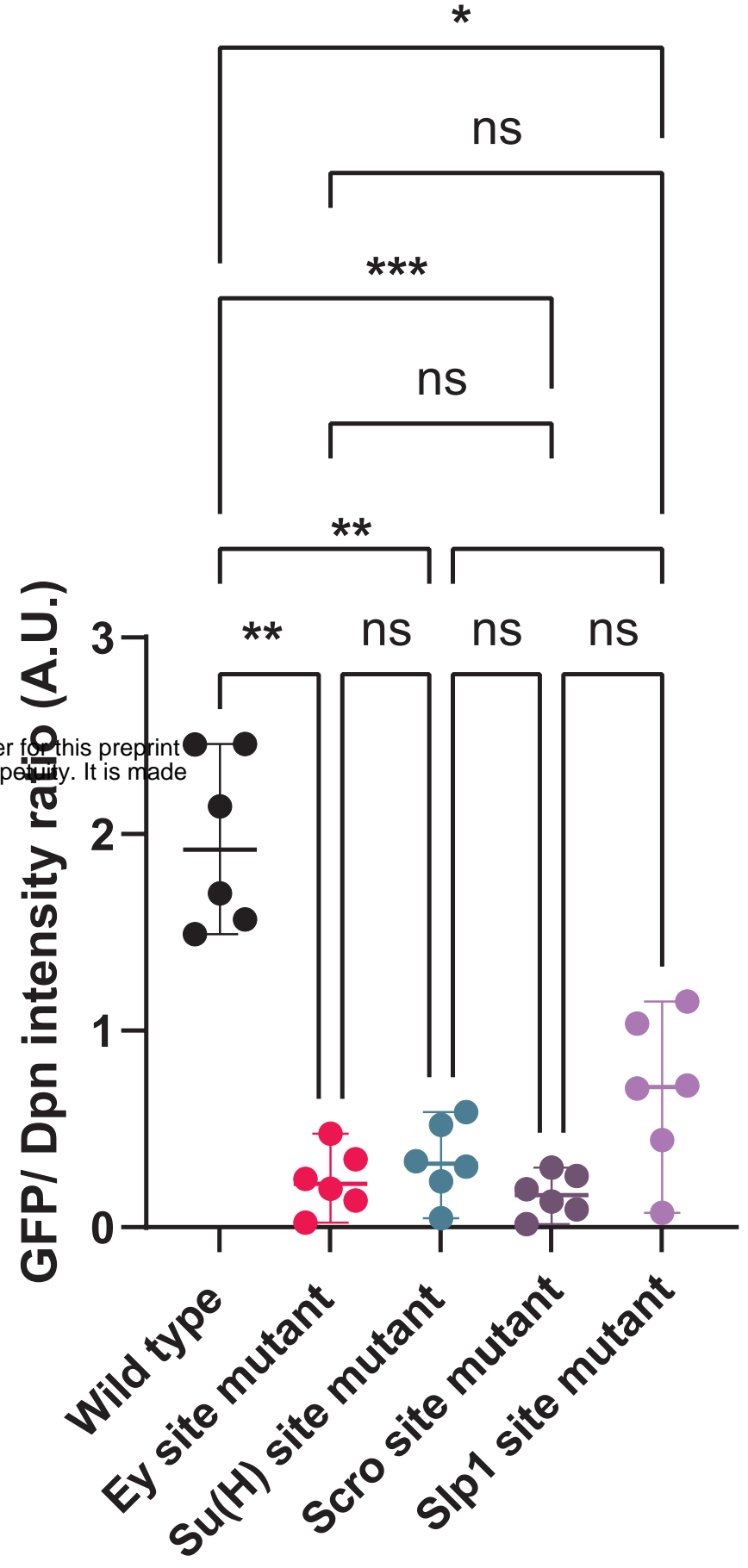


Figure 4. Mutations of putative binding sites for Ey, Su(H) and Scro in u8772 220 bp enhancer confirm their requirement for activating expression of SIp1/2 genes. Expression of GFP reporter driven by the wild-type 220 bp enhancer ( $\left.A-A^{\prime \prime \prime}\right)$ was compared against GFP reporters driven by mutated versions of the 220 bp enhancer in which predicted binding sites for specific transcription factors had been mutated: (B-B'") with predicted Ey binding sites mutated, (C-C'”) with predicted $\mathrm{Su}(\mathrm{H})$ binding sites mutated, (D- $\left.\mathrm{D}^{\prime \prime}\right)$ with predicted Scro binding sites mutated, (E-E'"') Slp1 binding sites mutated. Loss of binding sites for Ey, Su(H) and Scro in the $220 \mathrm{bp}$ enhancer led to a loss of GFP reporter expression. Scale bar: 20 microns. F. Intensity comparisons of mutated and wild type GFP reporters of the $48772220 \mathrm{bp}$ enhancer. The decrease in intensity of GFP expression for Ey, Scro, Su(H) and SIp1 binding site mutated reporters relative to wild type are all statistically significant (RM One-way ANOVA was performed with the Geisser-Greenhouse correction, $p$ value 0.0001 ; this was statistically significant, since $p<0.05$. Adjusted $p$ values from Tukey's multiple comparison test for mutants versus wild type are as follows: for Ey mutant $p=0.0013$, for $\mathrm{Su}(\mathrm{H})$ mutant $p=0.0032$, for Scro site mutant $p=0.0007$, for SIp1 site mutant $p=0.0225$. Six different sets of observations were used for quantification ( $n=6)$ ). Graphs indicate mean and 95\% C.I. and distributions of each data point about the mean. 
Figure 5

d5778 850 bp enhancer

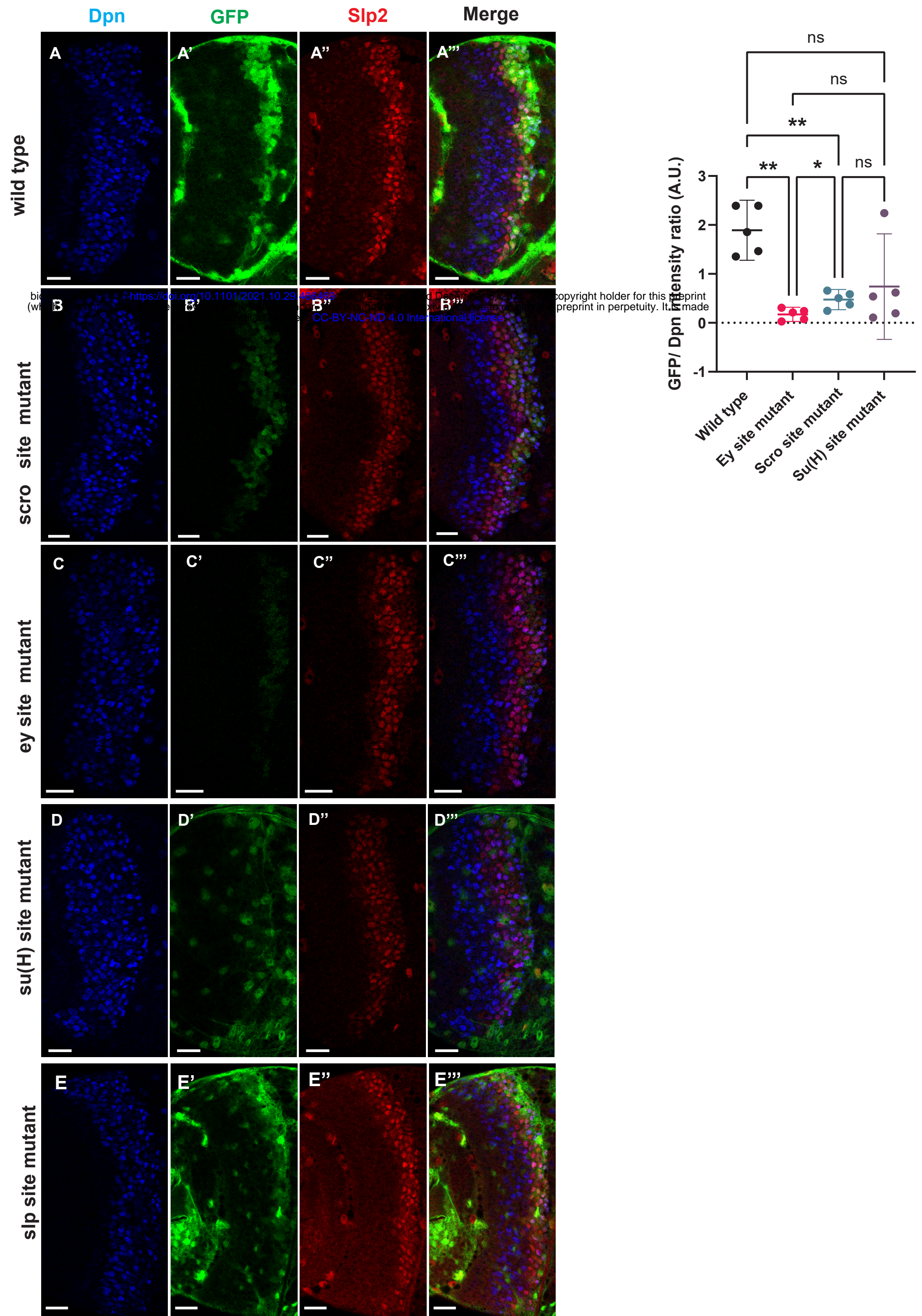


Figure 5. Mutations of putative binding sites for Ey, Su(H) and Scro in d5778 850 bp enhancer confirm their requirement for activating expressions of Slp1/2 genes. A parallel comparison was made for GFP reporter expression driven by the wild type 850 bp enhancer ( $\left.A-A^{\prime \prime \prime}\right)$ to the GFP reporter expression driven by versions of the $850 \mathrm{bp}$ enhancer with binding sites for specific transcription factors Scro, Ey, Su(H) and Slp1 mutated (B-B"', C-C'"', D-D'", and E-E'"' respectively). Loss of binding sites for Ey nearly obliterated GFP reporter expression in neuroblasts as did the loss of $\mathrm{Su}(\mathrm{H})$ binding sites. Additionally, in $\mathrm{Su}(\mathrm{H})$ site mutant expression of GFP reporter was up-regulated in surface glial cells. Loss of potential Scro binding sites also greatly reduced GFP reporter expression. Loss of SIp1 binding sites did not lead to a significant reduction in intensity (E-E'”). Scale bars: 20 microns. (F) Quantitation of d5778 850bp reporter intensities of the wild type and the factor binding site mutated variants shows that the reduction of GFP intensity compared to wild type for Ey binding site and Scro binding site mutated reporters is statistically significant (RM one-way ANOVA was performed with the Geisser-Greenhouse correction and $p$ value was calculated to be 0.005 ; this was statistically significant as $p<0.05$. Adjusted $p$ values from Tukey's multiple comparison test between wild type and mutants are as follows: for Ey site mutant $p=0.0027$, for Scro site mutant $p=0.0087$, for $\mathrm{Su}(\mathrm{H})$ site mutant $\mathrm{p}=0.1076$. Five different sets of observations were used for quantification $(n=5))$. For the $\mathrm{Su}(\mathrm{H})$ site mutated variant, though GFP expression was visibly reduced in neuroblasts, the unavoidable contributions of GFP signals from surface glial cells has confounded our measurements of GFP reporter intensity, making GFP signals appear stronger and the intensity reduction relative to wild type statistically insignificant. 


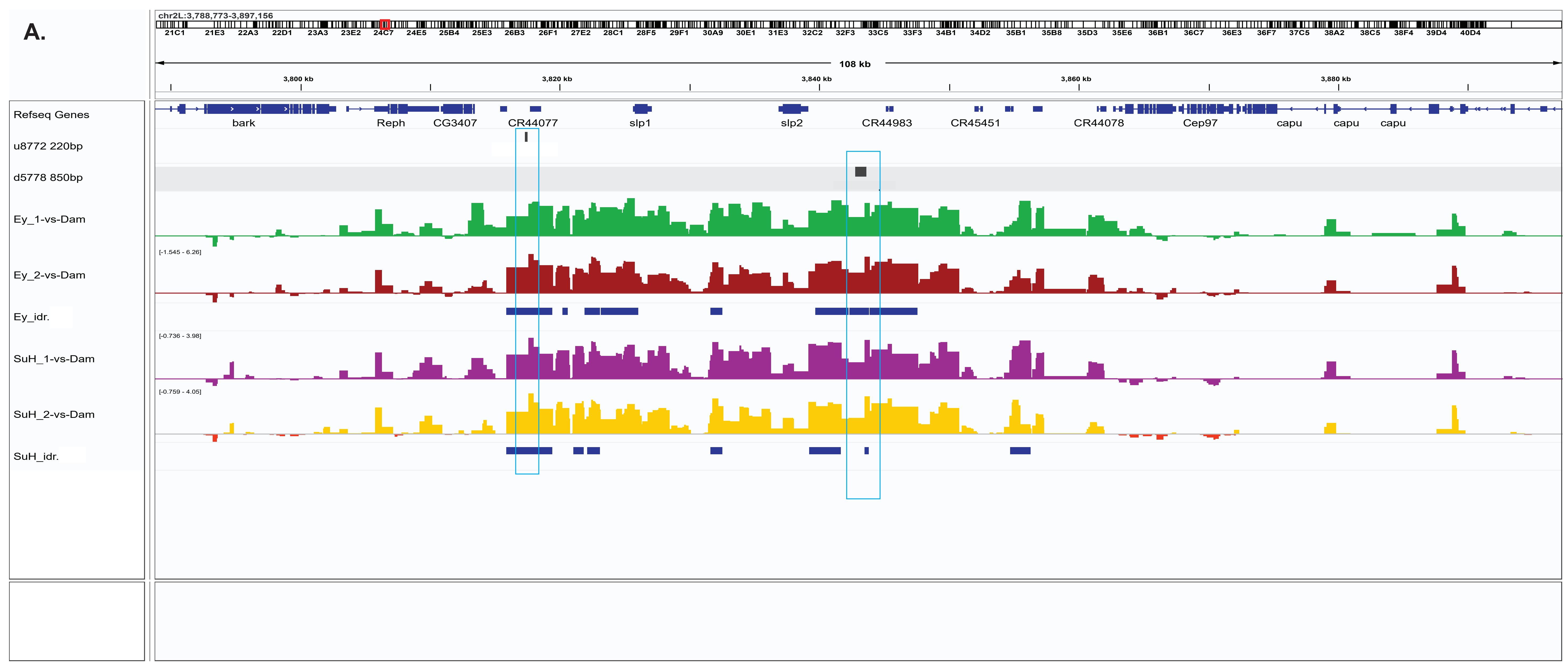

\begin{tabular}{|c|c|}
\hline $\begin{array}{l}\text { C.GO Term: Asymmetric Cell Division } \\
\text { CG3779-RB(numb) } \\
\text { G protein alpha i subunit(Galphai) } \\
\text { Notch(N) } \\
\text { brain tumor(brat) } \\
\text { frizzled(fz) } \\
\text { neuralized(neur) } \\
\text { prospero(pros) }\end{array}$ & $\begin{array}{l}\text { D. GO Term: Cell division } \\
\text { Cyclin A(CycA) } \\
\text { Cyclin E(CycE) } \\
\text { Cyclin G(CycG) } \\
\text { diaphanous(dia) } \\
\text { peanut(pnut) } \\
\text { pontin(pont) } \\
\text { string(stg) }\end{array}$ \\
\hline
\end{tabular}

E. GO Term:Apical Cell Polarity
Abl tyrosine kinase(Abl)
CG42684 gene product from transcript
CG42684-RB
G protein alpha i subunit(Galphai)
TNF-receptor-associated factor 4
(Traf4)
brain tumor(brat)
miranda(mira)
prospero(pros)

F. GO Term: Nuclear hormone receptor

Ecdysone receptor(EcR)

Ecdysone-induced protein 75B (Eip75B) knirps-like(knrl)
B. Overlap between Ey-Dam and Su(H)-Dam bound genes

\section{H. GO Term: Axon guidance}

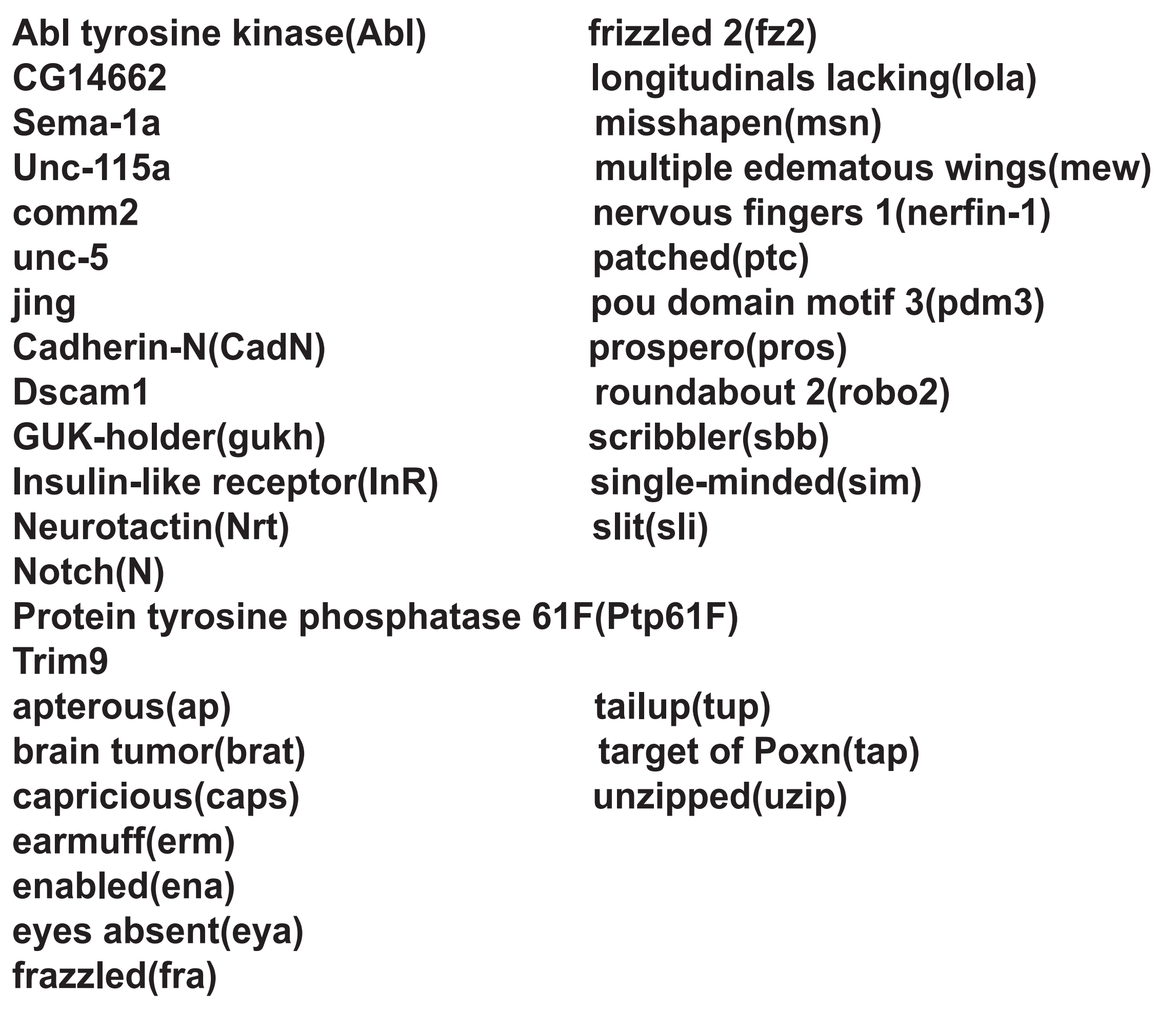

frizzled 2(fz2)

longitudinals lacking(lola)

nervous fingers 1 (nerfin-1)

prospero(pros)

scribbler(sbb)

single-minded(sim)

Neurotactin(Nrt)

Notch(N)

Trim9

apterous(ap)

target of Poxn(tap)

earmuff(erm)

eyes absent(eya)

\section{G. GO Term:Notch}

Abl tyrosine kinase(Abl)

Beadex(Bx)

Bearded(Brd)

numb

E(spl)m8-HLH

E(spl)mbeta-HLH

E(spl)mdelta-HLH

E(spl)mgamma-HLH

Epidermal growth factor receptor

Neurotactin(Nrt)

Serrate(Ser)

Star(S)

atonal(ato)

big brain(bib)

canoe(cno)

enabled(ena)

extra macrochaetae (emc)

fringe(fng)

frizzled(fz)

longitudinals lacking(lola)

neuralized(neur)

patched(ptc)

plexus(px)

pointed(pnt)

polychaetoid(pyd)

prickle(pk)

string(stg)

tribbles(trbl) 
Figure 6. Genome-wide binding of Ey and $\mathrm{Su}(\mathrm{H})$ were examined by DamID-seq. (A) Prominent peaks of Ey and $\mathrm{Su}(\mathrm{H})$ binding are seen in neighborhood of the s/p1 and s/p2 gene loci including at the genomic locations of identified $220 \mathrm{bp}$ and $850 \mathrm{bp}$ enhancers in both replicates of Ey-Dam and $\mathrm{Su}(\mathrm{H})$-Dam experiments. Peaks at the $\mathrm{u} 8772220 \mathrm{bp}$ and the $\mathrm{d} 5778$ 850bp enhancers have passed IDR< 0.05 cut-off supporting their reproducibility. (B) Output from BioVenn showing overlap between genes bound by EyDam and Su(H)Dam suggesting that Ey may act as a priming factor in recruiting $\mathrm{Su}(\mathrm{H})$ to enhancers of Notch-target genes in neuroblasts. (C-H) GO terms associated with genes that are bound by both Ey and $\mathrm{Su}(\mathrm{H})$ and thus may be co-regulated by Ey and the Notch pathway. Genes involved in a variety of processes relevant to neuroblasts and neural differentiation are seen. 

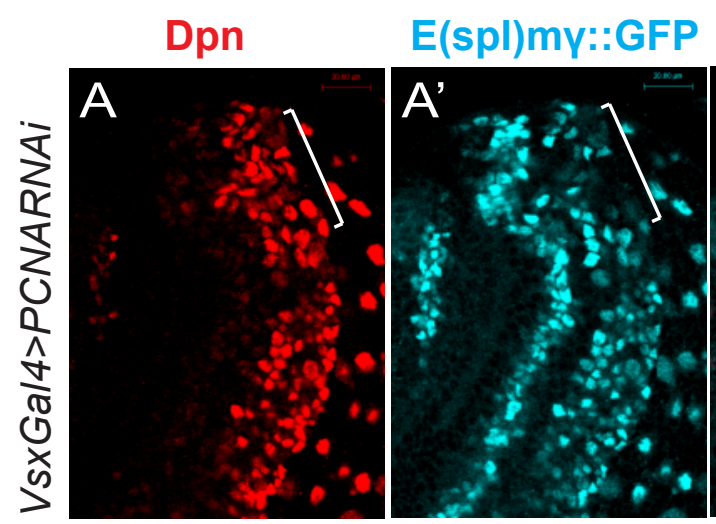

Merge

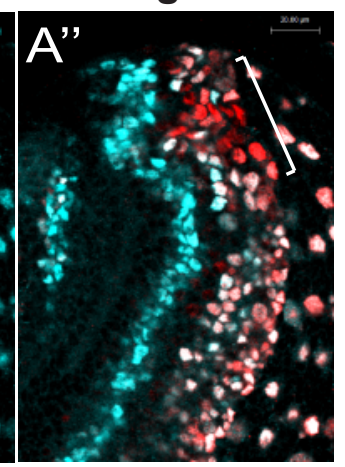

Ey
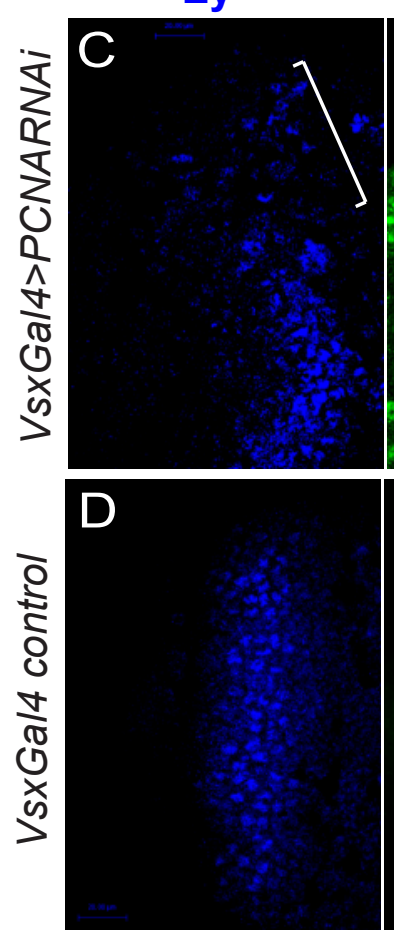
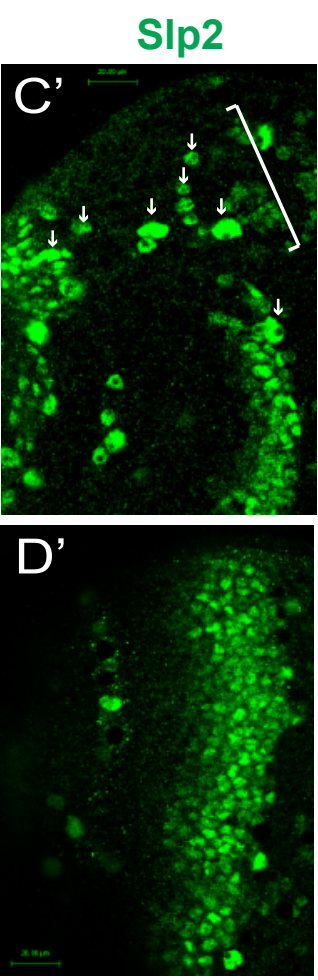

Ey SIp2 Dpn
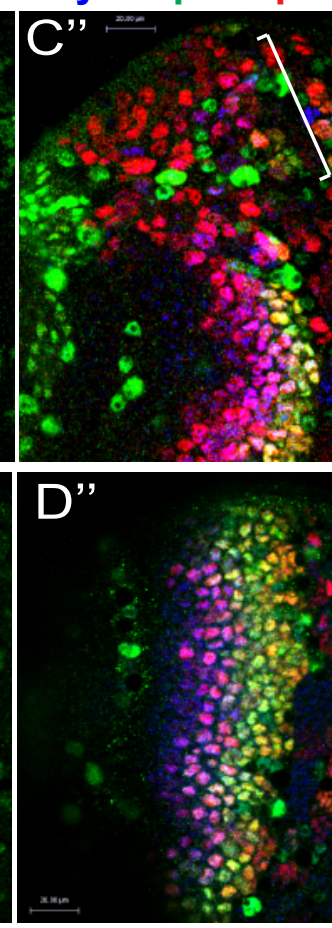

Dpn

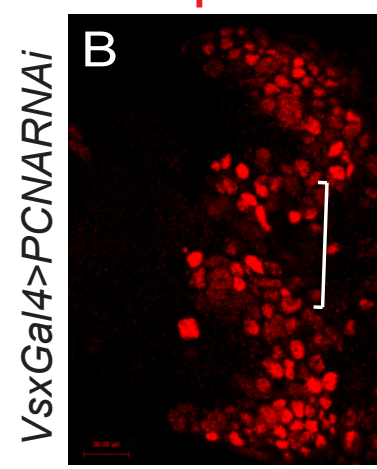

SoxN
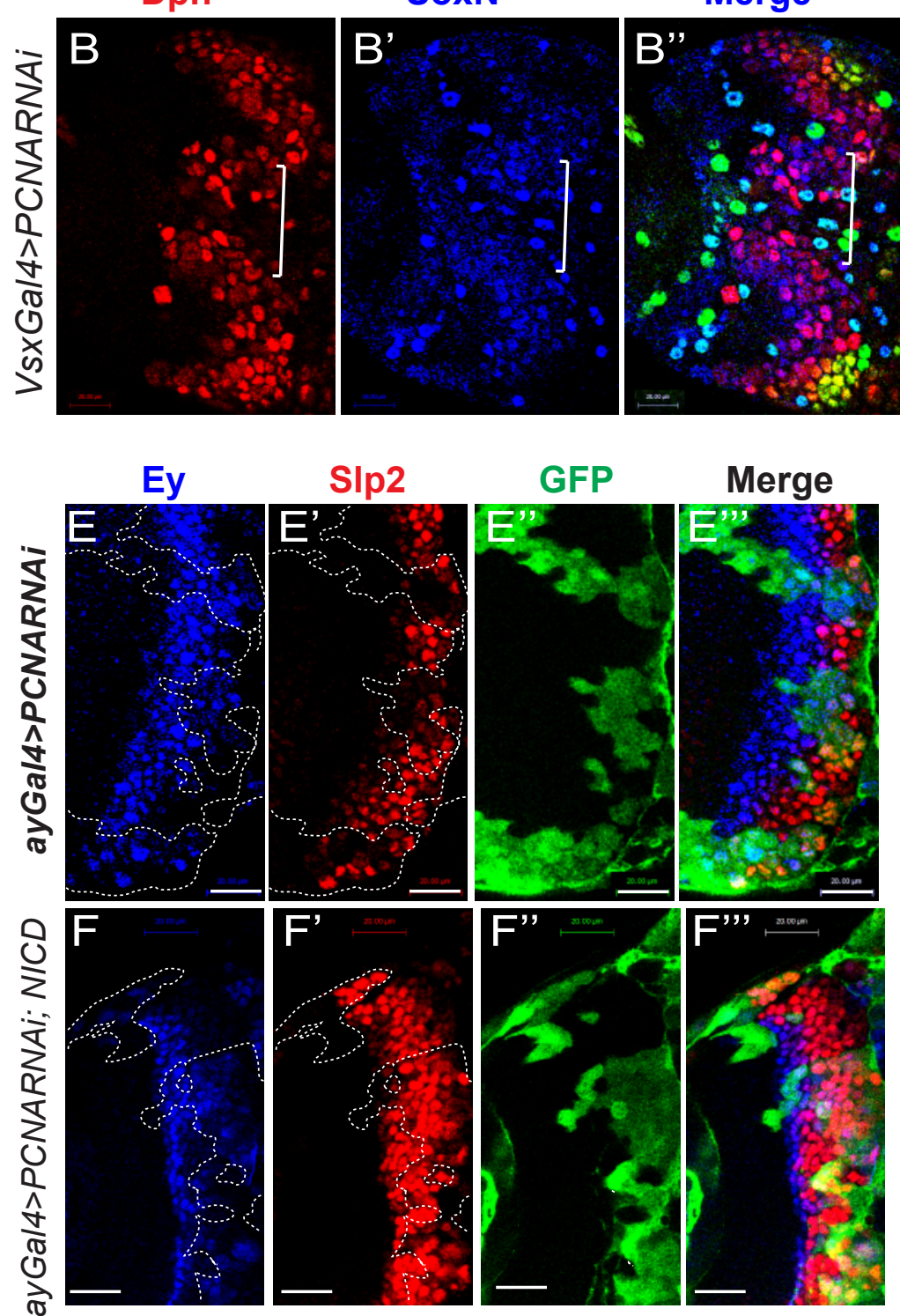

SIp2
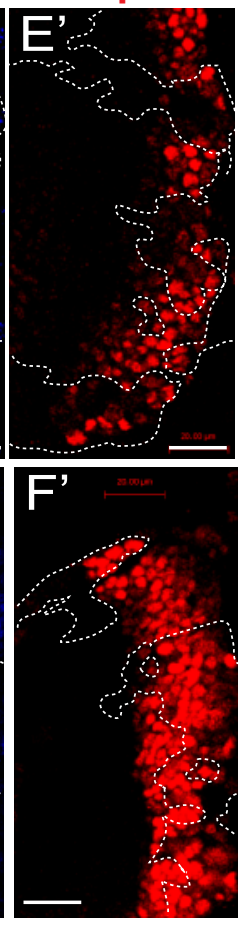

GFP
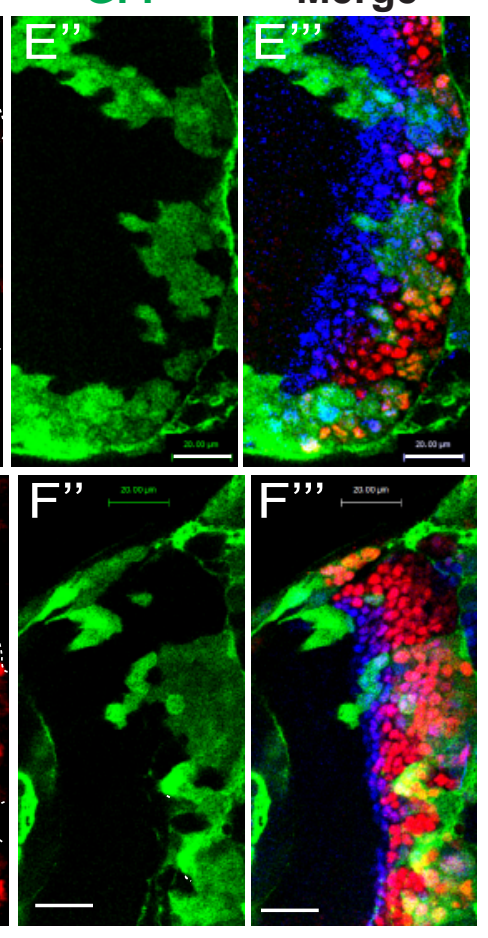

Figure 7. Cell cycle progression is required for the precise timing of SIp expression and this is mediated in part through Notch signaling.

(A-A'") The expression of Dpn (red) and E(spl)myGFP (cyan) in vsxGal4 driving UASDCR2 and UAS-PCNARNAi (VDRC51253) optic lobes $(n=5)$. The affected domain is indicated by a bracket. (B-B'") The expression of Dpn (red) and SoxN (blue) in vsxGal4 driving UASDCR2 and UASPCNARNAi optic lobes ( $n=5$ ). (C-C') The expression of Dpn (red), Ey (blue) and Slp2 (green) in vsxGal4 driving UASDCR2 and UAS-PCNARNAi optic lobes $(n=9)$. The affected domain is indicated by a bracket. Some glia express Slp2 but not Dpn are indicated by white arrows. (D$D^{\prime \prime}$ ) The expression of Dpn (red), Ey (blue) and SIp2 (green) in vsxGal4; UASDCR2 control optic lobes. (E-E'") Larvae of genotype ywhsFLP; ayGal4UASGFP /UAS-PCNARNAi; UASDCR2/+ were heat shocked at $37 \mathrm{C}$ for $8 \mathrm{~min} 70$ hours before dissection at $3^{\text {rd }}$ instar larval stage. Ey expression (blue) is minimally affected in clones marked by GFP (green), while SIp2 (red) expression is delayed (17 clones). (F-F"') Larvae of genotype ywhsFLP; ayGal4UASGFP /UAS-PCNARNAi; UASDCR2/dpn>FRT-STOP-FRT-NICD were heat shocked at 37C for $8 \mathrm{~min} 70$ hours before dissection at $3^{\text {rd }}$ instar larval stage. Ey expression (blue) is minimally affected in clones marked by GFP (green), while SIp2 (red) expression initiation is rescued (10 clones). Scale bar 20 microns. 

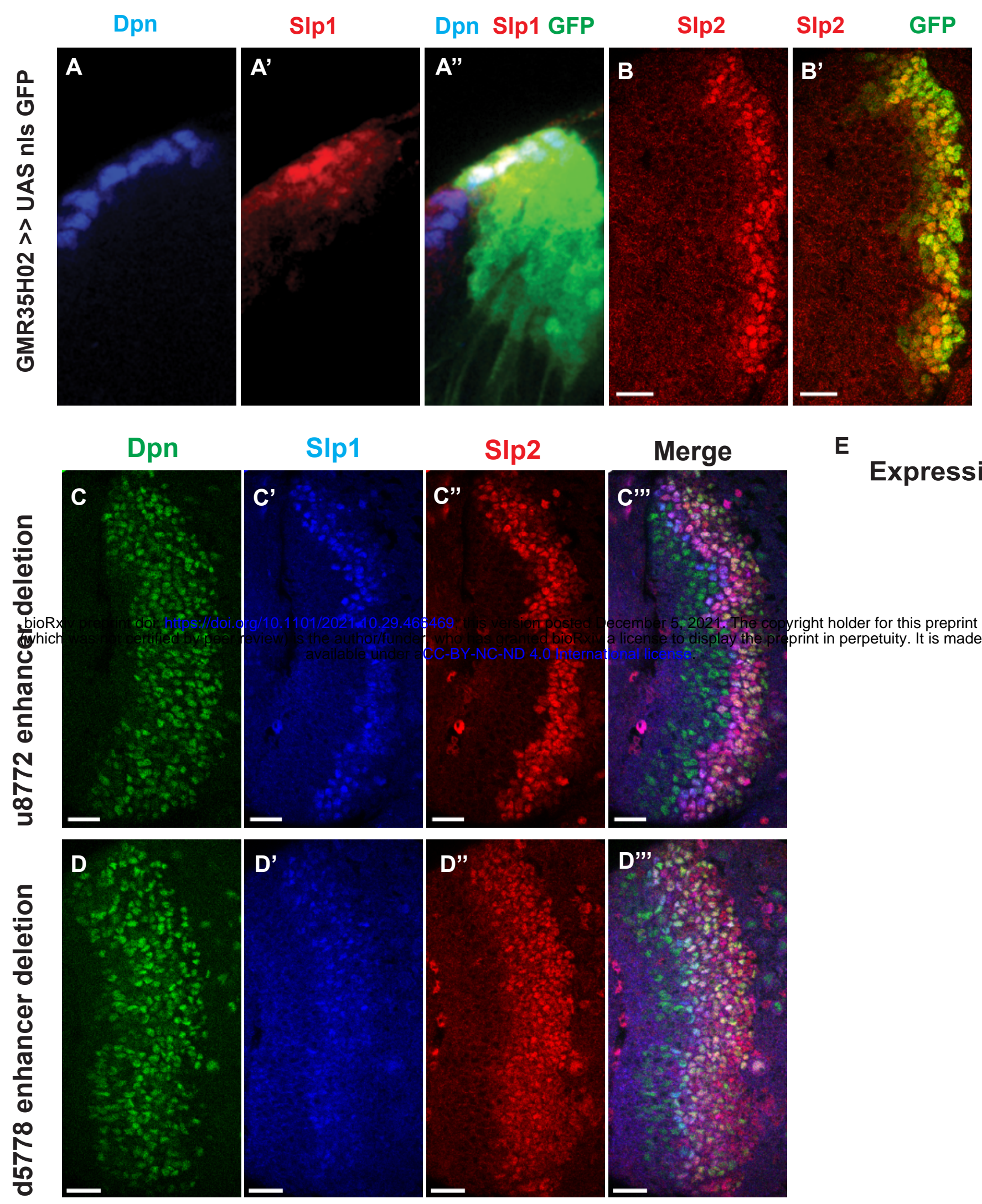

E Expression of SIp1 (normalized to brain size)

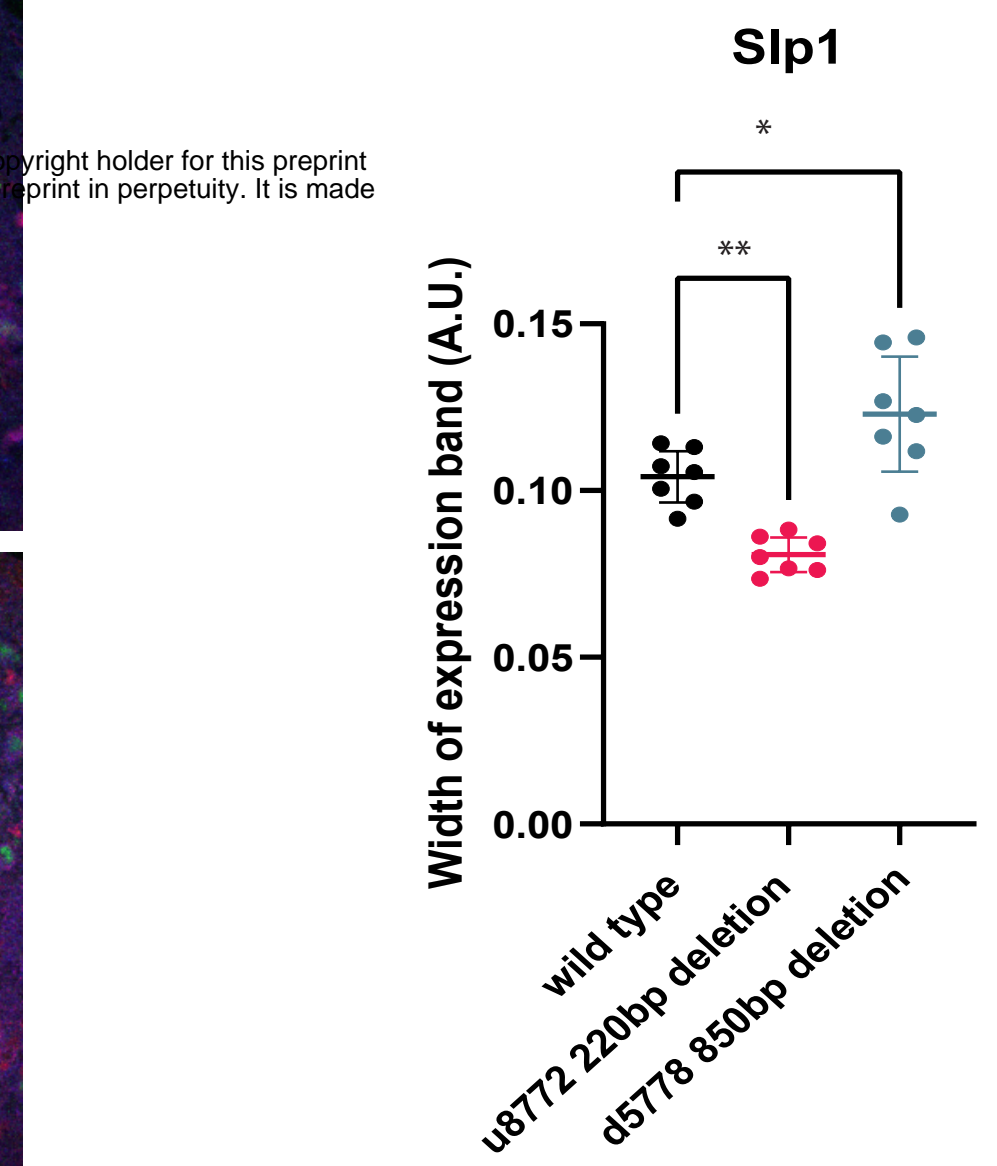

F Expression of SIp2 (normalized to brain size)

G Expression of Dpn (normalized to brain size)
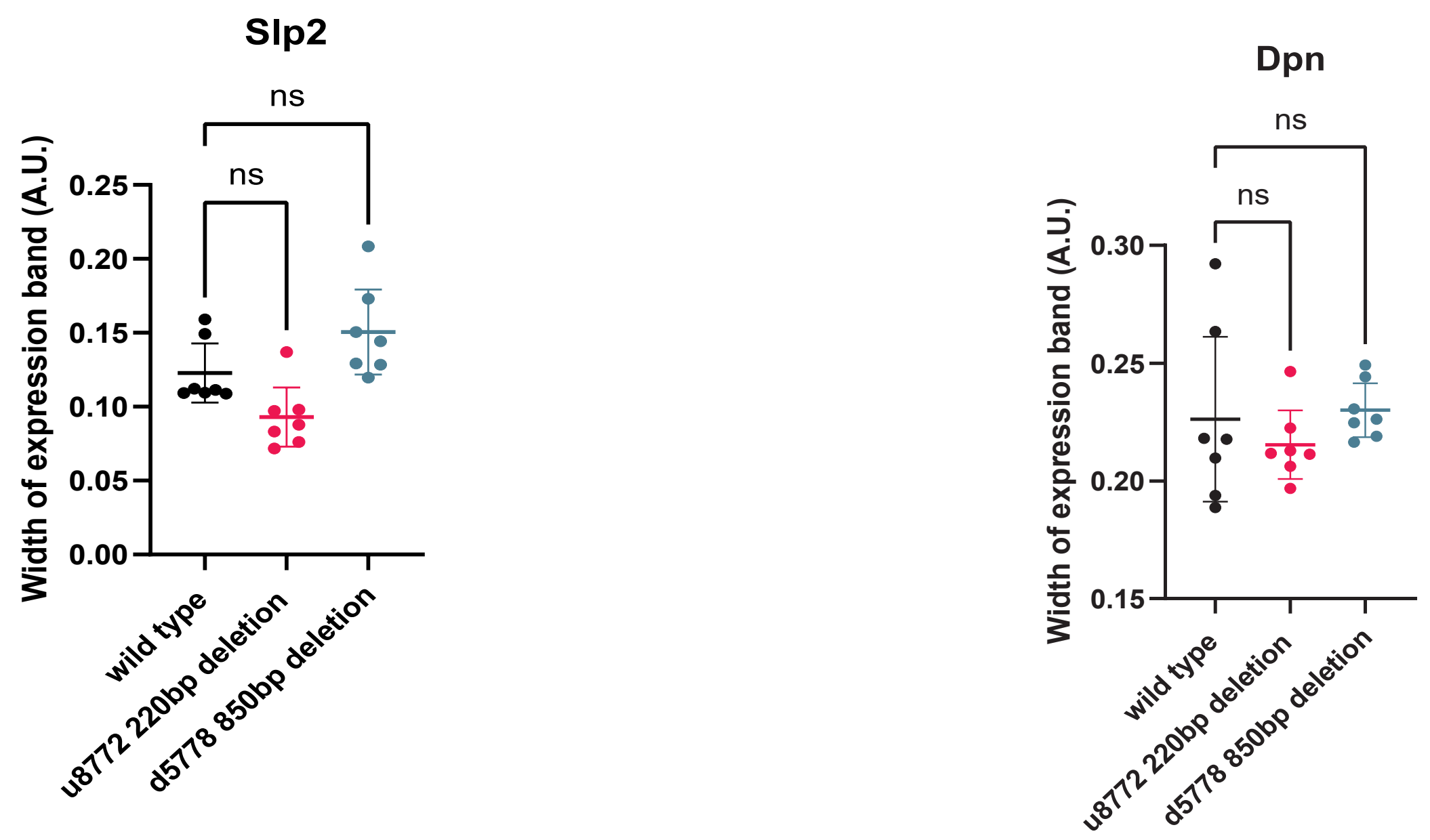
Supplementary Figure S1. SIp1/2 expression is regulated by at least two enhancers. (A-A'"') UAS-GFP driven by GMR35H02-Gal4 is initiated the same time as endogenous SIp1 in neuroblasts marked by Dpn. This is showing a cross-section view. (B-B') UAS-GFP driven by GMR35H02-Gal4 perfectly overlaps with SIp2 expression. (C-D'"') CRISPR-Cas9 deletions of the u8772 enhancer (C- $\left.C^{\prime \prime \prime}\right)$ and the d5778 enhancer (D- $\left.D^{\prime \prime \prime}\right)$ individually do not abolish SIp1/2 expression suggesting possible redundance. Scale bars: 20 microns. (E) Comparison of widths of expression domains normalized to brain size for SIp1 in wild type and enhancer deleted brains. $P$ value of ordinary one way ANOVA is $<0.0001$ and is statistically significant because $p<0.05$. Adjusted $p$ values from Dunnett's multiple comparison test between wild type and the $u 8772$ $220 \mathrm{bp}$ enhancer deletion is 0.0041 , between wild type and d5778 $850 \mathrm{bp}$ enhancer deletion is 0.0187 . Both are statistically significant since $p<0.05$. Data from seven brains of each genotype is quantified $(n=7)$. (F) Expression domain width normalized to brain size for SIp2 compared between wild type and enhancer deleted brains shows no statistically significant differences relative to wild type ( $p$ value from ordinary one way ANOVA $=0.0019$, statistically significant because $p<0.05$. Adjusted $p$ values from Dunnett's multiple comparison test relative to wild type are as follows: for u8772 220bp enhancer deletion $p=0.0730$, for $d 5778$ 850bp enhancer deletion $p=0.0965$. Neither are statistically significant since $p>0.05)$. Data from seven brains of each genotype is quantified ( $n=7)$. (G) Expression domain width normalized to brain size for Dpn also shows no significant differences between wild type and enhancer deletion mutants ( $p$ value from ordinary one-way ANOVA is 0.5277 and is $>0.05$, hence not statistically significant. Adjusted $p$ values from Dunnett's multiple comparisons test between enhancer deletion mutants and the wild type are as follows: for the $48772220 \mathrm{bp}$ enhancer deletion $p=0.6310$, for the $d 5778$ 850bp enhancer deletion $p=0.9414$. Neither are statistically significant being $>0.05$.) Data from seven brains of each genotype is quantified $(n=7)$. This shows that deletion of the enhancers doesn't affect Dpn expression. 

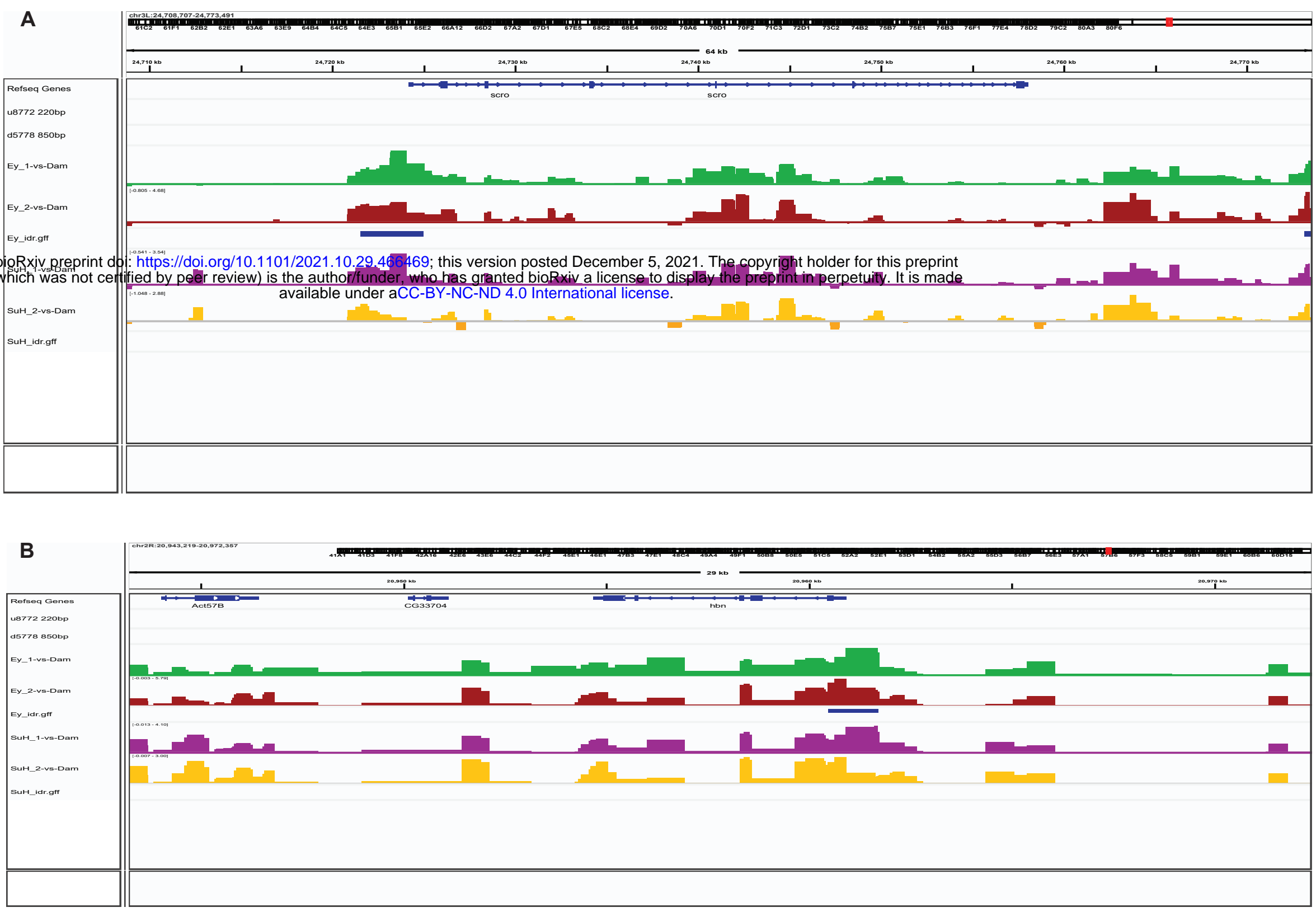

E

C

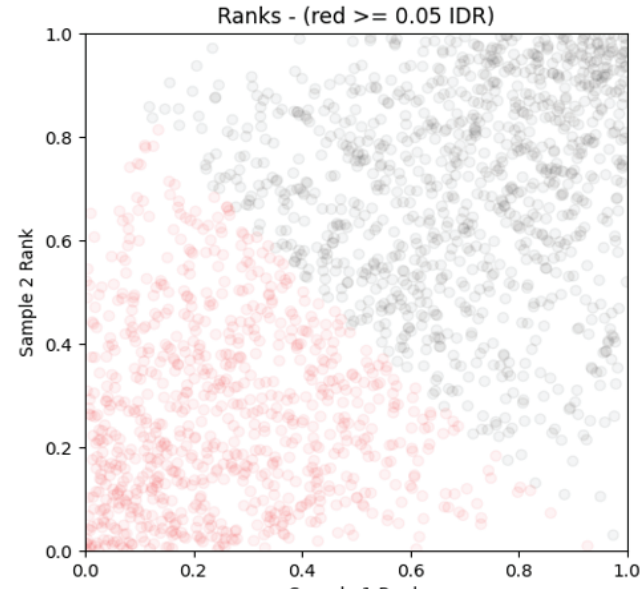

D

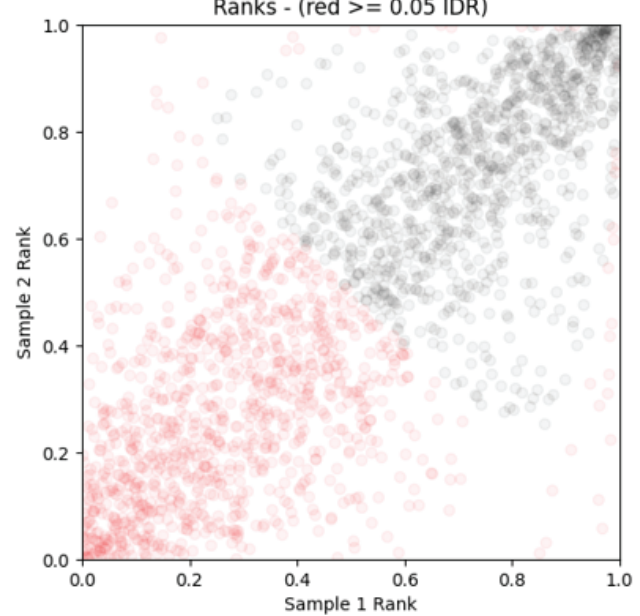

Log10 Scores - (red $>=0.05$ IDR)

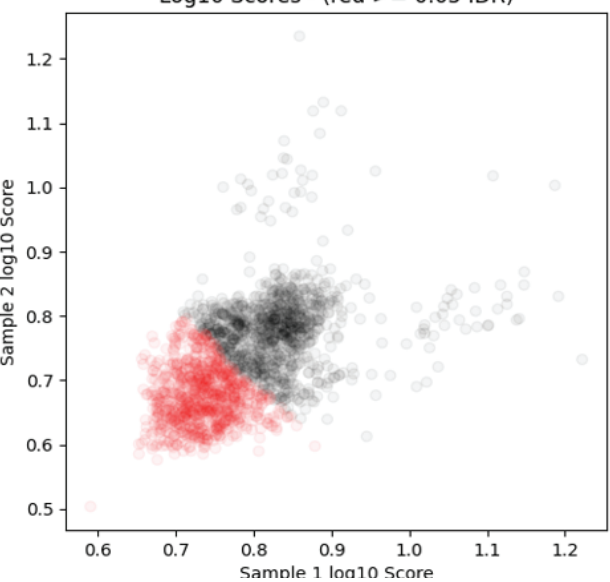

Log10 Scores - (red > $=0.05$ IDR)

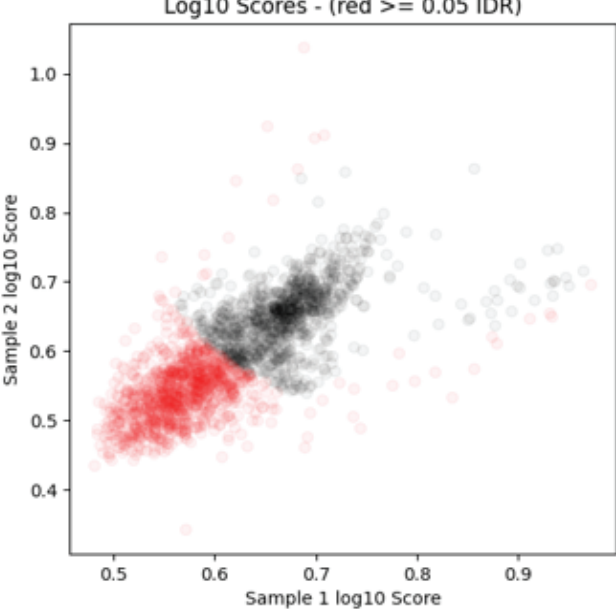

\begin{tabular}{|c|c|c|c|}
\hline $\begin{array}{l}\text { Transcription } \\
\text { Factor }\end{array}$ & $\begin{array}{l}\text { Number of } \\
\text { peaks }\end{array}$ & $\begin{array}{l}\text { Number of } \\
\text { peaks passing } \\
\text { IDR }<0.05\end{array}$ & $\begin{array}{l}\text { \% of peaks } \\
\text { passing IDR } \\
<0.05\end{array}$ \\
\hline Ey-Dam & 1810 & 984 & $54.4 \%$ \\
\hline Su(H)-Dam & 1996 & 972 & $48.7 \%$ \\
\hline
\end{tabular}




\section{Supplementary Figure S2. DamID-sequencing reveals Ey and Su(H) binding to other TTF} genes. $(A, B)$ DamID-seq also confirms EyDam and Su(H)Dam binding to scro $(A)$ and $h b n(B)$ loci that have been suggested by other studies to function as TTFs in optic lobe medulla neuroblasts. (C,D) Scatter plots from I.D.R. analysis of Ey-Dam (C) and Su(H)-Dam peaks (D) show reproducible peaks. Peaks represented by black dots are better reproducible and pass the I.D.R $<0.05$ threshold. (E) Summarized table of results from IDR analysis of Ey-Dam and Su(H)Dam. 


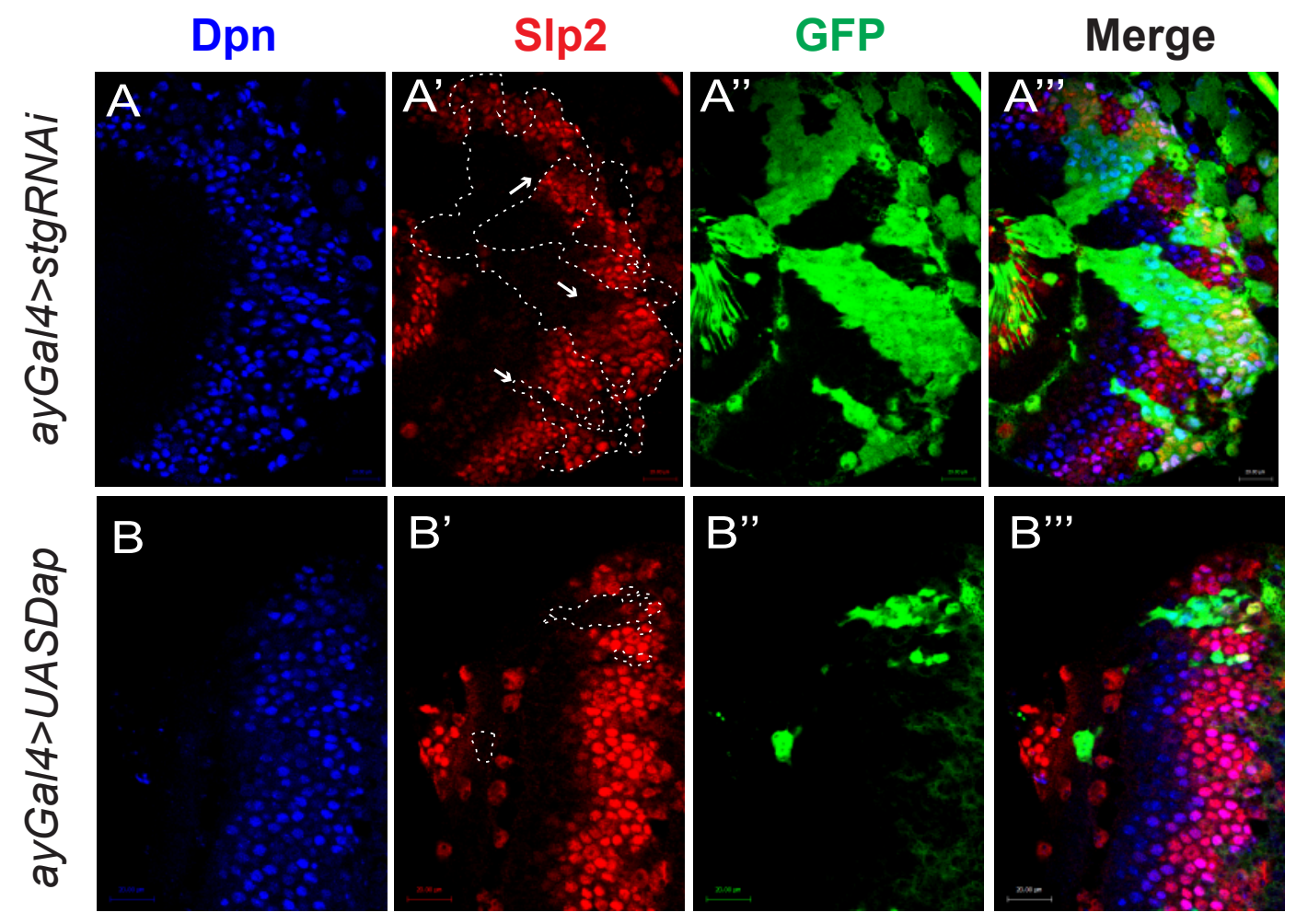

Supplementary Figure S3. Cell cycle progression is required for the temporal cascade progression. (A-A"') Larvae of genotype ywhsFLP; ayGal4UASGFP /+; UASDCR2/UASstgRNAi (VDRC 17760) were heat shocked at 37C for $8 \mathrm{~min} 70$ hours before dissection at $3^{\text {rd }}$ instar larval stage. Dpn expression (blue) is normal in clones marked by GFP (green), while Slp2 (red) expression is delayed (11 clones). (B-B"')' Larvae of genotype ywhsFLP; ayGal4UASGFP /+; UASDCR2/UAS-Dap (BDSC 83338) were heat shocked at 37C for $8 \mathrm{~min} 70$ hours before dissection at $3^{\text {rd }}$ instar larval stage. Dpn expression (blue) is normal in clones marked by GFP (green), while SIp2 (red) expression is delayed (8 clones). 